\title{
Sequential and Incremental Precoder Design for Joint Transmission Network MIMO Systems With Imperfect Backhaul
}

\author{
Ming Ding, Member, IEEE, Jun Zou, Zeng Yang, Hanwen Luo, and Wen Chen, Senior Member, IEEE
}

\begin{abstract}
In this paper, we propose a sequential and incremental precoder design for downlink joint transmission (JT) network multiple-input-multiple-output (MIMO) systems with imperfect backhaul links. The objective of our design is to minimize the maximum of the substream mean square errors, which dominates the average bit error rate (BER) performance of the system. In the proposed scheme, we first optimize the precoder at the serving base station (BS) and then sequentially optimize the precoders of nonserving BSs in the JT set according to the descending order of their probabilities of participating in the JT. The BS-wise sequential optimization process can improve system performance when some BSs have to temporarily quit the JT operations because of poor instant backhaul conditions. In addition, the precoder of an additional BS is derived in an incremental way, i.e., the sequentially optimized precoders of the previous BSs are fixed, and thus, the additional precoder plays an incremental part in multi-BS JT operations. An iterative algorithm is designed to jointly optimize the substream precoder and substream power allocation for each additional BS in the proposed sequential and incremental optimization scheme. Simulations show that, under the practical backhaul link conditions, our scheme significantly outperforms the autonomous global precoding scheme in terms of BER performance.
\end{abstract}

Index Terms - Imperfect Backhaul, joint transmission (JT), network multiple-input multiple-output (MIMO), precoding.

\section{INTRODUCTION}

$\mathbf{R}$ ECENTLY, there have been considerable interests in network multiple-input-multiple-output (MIMO) systems [1], where multiple geographically distributed multiantenna base stations (BSs) cooperate with each other to transmit data to users. In [2], it is shown that network MIMO can be employed to mitigate cochannel interference and exploit macrodiversity. Motivated by these works, downlink network MIMO technologies have been adopted by Fourth-Generation mobile communication standards, such as the Third-Generation

Manuscript received August 22, 2011; revised January 10, 2012 and March 15, 2012; accepted March 25, 2012. Date of publication April 20, 2012; date of current version July 10, 2012. This work was supported by Sharp Laboratories of China Co., Ltd. The review of this paper was coordinated by Prof./Dr. Y. Su.

M. Ding is with the Department of Electronic Engineering, Shanghai Jiaotong University, Shanghai 200240, China, and also with Sharp Laboratories of China Co., Ltd., Shanghai 201203, China (e-mail: dm2007@sjtu.edu.cn; ming.ding@cn.sharp-world.com).

J. Zou, H. Luo, and W. Chen are with the Department of Electronic Engineering, Shanghai Jiaotong University, Shanghai 200240, China (e-mail: zoujun@sjtu.edu.cn; hwluo@sjtu.edu.cn; wenchen@sjtu.edu.cn).

Z. Yang is with Sharp Laboratories of China Co., Ltd., Shanghai 201203, China (e-mail: zeng.yangcn.sharp-world.com).

Digital Object Identifier 10.1109/TVT.2012.2195690
Partnership Project (3GPP) Long Term Evolution-Advanced (LTE-A) networks [3].

The cooperating strategies for network MIMO systems can generally be divided into two categories, i.e., coordinated beamforming (CB) and joint transmission (JT). When the CB strategy is employed, the cooperating BSs share channel state information (CSI) in various forms, and each BS only transmits data to its own served users, whereas for the JT strategy, both CSI and user data should be shared by all cooperating BSs, and thus, each user receives data from multiple BSs. Much current research focuses on CB network MIMO schemes [4][7]. However, the performance of the $\mathrm{CB}$ strategy is generally interference limited due to the lack of abundant spatialdomain degrees of freedom for perfect inter-BS interference coordination in practical systems [1]. On the other hand, the JT strategy takes a more aggressive approach to cope with the interference problem by transforming the interference from neighbor BSs into useful signals. Usually, the mathematical form of the JT scheme bears a close resemblance to conventional MIMO systems except for its distributed structure [8]. From uplink-downlink duality theory, the capacity region of the downlink JT network MIMO systems can be computed from its dual uplink [9] with the same sum power constraint. These results were later generalized to accommodate the perantenna power constraint [10] by showing that the per-antenna downlink transmitter optimization problem can be transformed into a dual uplink problem with uncertain noise. It should be noted that most capacity duality results are based on nonlinear signal processing at the BS side, such as the dirty paper coding [11], which is computationally demanding for precoding across multiple BSs. This has motivated research in linear precoding for JT, such as zero-forcing precoders [12], which are much more easy to implement compared with the dirty paper coding precoder. Moreover, when the user equipment (UE) is equipped with multiple antennas, the distributed transceiver design [13], i.e., designing the precoder with UE's receiver structure taken into account, should also be considered for JT. Another relevant issue regarding JT are the imperfect backhaul links [14]. In practice, cooperating BSs are connected through imperfect links with finite capacity, unpredictable latency, and limited connectivity. For example, the latency of practical backhaul links such as the copper and wireless interface varies from several milliseconds to tens of milliseconds, depending on the technology/standard. Moreover, when the backhaul communication is based on a generic IP network, the backhaul latency also depends on the number of routers between two 
cooperative BSs and the topology of the network, e.g., star, ring, tree, mesh, etc. Furthermore, congestion in the routers causes an extra delay, typically several milliseconds [15]. It should be noted that limited capacity is another important backhaul issue [16]. Most of the current cellular backhaul networks are designed for handover functions, which are not suited for data exchange in a large amount [3]. Constraints from lower capacity/higher latency backhaul communication in coordinated multipoint operations were studied in the $3 \mathrm{GPP}$ LTE-A meetings [17], [18]. Due to the immature status of the study, remote radio head or remote radio equipment based centralized BS and fiber based backhaul [19] were assumed for the JT as a starting point of the working order for the imperfect backhaul issue [20]. Although the current centralized network structure and fiber based backhaul will not pose serious problems for existing JT schemes, for future JT operations, the impact of imperfect backhaul should be carefully investigated [21]. Up to now, theoretical performance bounds for JT network MIMO with unreliable backhaul links among the cooperating BSs are still unknown [1]. Finally, it should also be noted that imperfect CSI fed back by the UE is a common assumption in practical frequency-division-duplexing (FDD) systems such as the 3GPP LTE-A system, where the downlink CSI cannot be inferred from the uplink CSI. For imperfect CSI feedback in a practical system, implicit CSI feedback, i.e., feedback of precoder recommendation by UEs [3], is much more preferred than explicit CSI feedback, i.e., feedback of the channel matrix, due to feedback overhead considerations. In this paper, we consider the implicit CSI feedback, which has been widely adopted by practical systems such as the 3GPP LTE-A system [3].

In this paper, we investigate the precoder design for downlink JT network MIMO systems with imperfect backhaul links, i.e., finite capacity, unpredictable latency, and limited connectivity. In particular, we focus on network impairments incurred by backhaul delays. For JT operations, both the transmission data and the CSI must be available at the actual transmission BSs before the transmission starts; otherwise, the JT cannot be operated as it was supposed to be. In a multi-BS JT network where one serving BS and several helper BSs constitute a JT set, we assume that the UE's data will always arrive at the serving BS from higher-layer entities in a timely and error-free manner, and then the serving BS shares the data with the helper BSs by means of imperfect backhaul communications. Here, the serving BS does not necessarily mean the BS with the strongest signal level at the UE, the typical event of which occurs during inter-BS handover process. According to [3], the serving BS is defined as the BS sending downlink control signalings, e.g., downlink scheduling information, to the UE. In the sense of downlink control signaling connection, the helper BSs are not equal partners with the serving BS because they only provide data transmissions to the UE. In addition, regarding CSI feedback, we assume that the UE reports the precoder recommendation information either to each individual BS in the JT set or to the serving BS that in turn exchanges this information among helper BSs over backhaul links. Whether both the transmission data and the CSI arrive at a certain helper BS before the JT scheduled to be performed or not is a probabilistic event because of the nondeterministic delay, which has been shown to conform to a shifted gamma distribution in [22]. If a helper BS fails to obtain both the transmission data and the CSI in time, then it has to quit the JT operations. Thereby, whether a helper BS can participate in JT or not is also a probabilistic event. Since the average bit error rate (BER) of the system is generally dominated by the substream with the maximum mean square error (MSE) [23], we propose a precoding scheme to minimize the maximum of the substream MSEs. In the proposed scheme, we first optimize the precoder at the serving BS and then sequentially optimize the precoders of helper BSs in the JT set according to the descending order of their probabilities of participating in JT. The BS-wise sequential optimization process can improve the system performance when some helper BSs have to temporarily quit the JT operations because of poor instant backhaul conditions. In addition, the precoder of an additional BS is derived in an incremental way, i.e., the sequentially optimized precoders of the previous BSs are fixed; thus, the additional precoder plays an incremental part in the multi-BS JT operations. Because the BS precoders are generated sequentially and incrementally, our proposed scheme will be referred to as the sequential and incremental precoding (SIP) scheme hereafter. An iterative algorithm is designed to jointly optimize the substream precoder and power allocation (PA) for each additional BS in the SIP scheme. Simulation results show that our scheme can achieve considerable gains in terms of BER performance compared with a variation of the global precoding (GP) scheme, i.e., autonomous GP (AGP) scheme, under practical backhaul link conditions.

There are several benefits offered by our scheme. First, it offers flexibility to the JT network MIMO since the helper BSs can adaptively decide whether to join JT according to their own situation. Such JT scheme enables the network MIMO to adaptively switch among single BS transmission (ST), partial JT, and full JT without inter-BS signaling. Here, partial JT refers to the transmission from a subset of BSs within the JT set. Second, the related CSI feedback scheme can easily fit into the current 3GPP LTE-A per-BS feedback framework [3], i.e., the feedback operation is performed on a per-BS basis, which facilitates the feedback design. Third, the complexity on the UE side to select a preferred precoder from a codebook is low. Instead of searching for the precoder through a large codebook, as done in the conventional GP scheme, the precoder for each BS is obtained from a small per-BS-based codebook.

The rest of this paper is organized as follows. Section II presents the system model and briefly describes backhaul impairments. Section III discusses the GP and AGP schemes. Section IV proposes the sequential and incremental precoder design and its extension to multi-BS JT network MIMO systems. This paper is completed with simulation results and conclusions in Sections V and VI, respectively.

Notations: $(\cdot)^{\mathrm{T}},(\cdot)^{*},(\cdot)^{\mathrm{H}}$, and $\operatorname{tr}\{\cdot\}$ stand for transpose, conjugate, conjugate transpose, and trace of a matrix, respectively. $\mathbf{A}_{i, j}, \mathbf{A}_{i,:}$, and $\mathbf{A}_{:, j}$ denote the $(i, j)$ th entry, $i$ th row, and $j$ th column of matrix $\mathbf{A}$, respectively. $\operatorname{diag}\left\{\mathbf{A}_{k}\right\}$ denotes a block-diagonal matrix with the $k$ th diagonal block given by $\mathbf{A}_{k} . \mathbf{I}_{N}$ stands for an $N \times N$ identity matrix. $|\mathbf{a}|$ denotes the Euclidean norm of a vector a. $\mathbb{E}\{\cdot\}$ and $\operatorname{Re}\{\cdot\}$ denote 


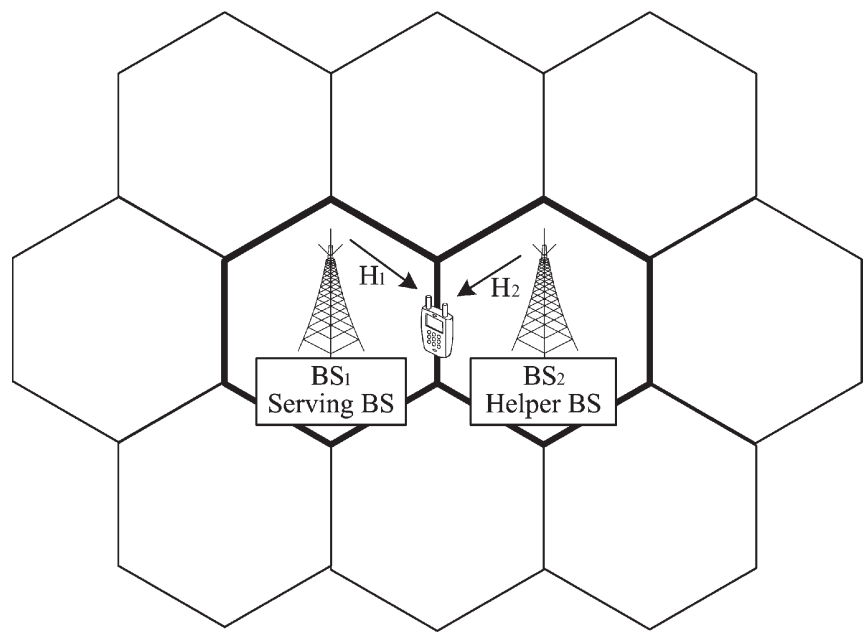

Fig. 1. Two-BS JT network.

expectation operator and the real part of a complex value, respectively. Finally, we define $(a)^{+}=\max (0, a)$.

\section{SYSTEM MODEL}

In this section, we address the system model and briefly discuss backhaul impairments. We consider a multicell wireless network consisting of $B$ adjacent $\mathrm{BSs}$, where each BS is equipped with $N_{T}$ antennas. A cell edge UE with $N_{R}$ antennas is served by one serving $\mathrm{BS}$, and the other $B-1 \mathrm{BSs}$ are helper BSs that adaptively provide service to the UE, depending on the backhaul conditions. In practice, the candidates of helper BSs can be decided either by UE based on received reference signal strength of nearby BSs or by the serving BS based on wideband CSI reported by UE. The selection algorithm to decide the helper BSs can be found in [24] and [25]. Here, we assume $B-1$ helper BSs have already been selected based on some existing BS selection schemes. Moreover, in practical scenarios, values of $B$ are relatively small, usually not larger than 4 [26].

\section{A. JT Networks With Two BSs}

Our basic idea is to optimize the precoders for the $B$ BSs in the JT set to derive per-BS precoders one by one in a sequential and incremental manner. Hence, the most basic scenario is a JT network with only two BSs. A two-BS JT network $(B=2)$ is shown in Fig. 1, which serves as an instructive example to formulate the key problem of our concern. An $N_{R}$-antenna cell edge $\mathrm{UE}$ is associated with a serving $\mathrm{BS}_{1}$, and the transmission is assisted by a helper $\mathrm{BS}_{2}$. Note that the results from this model will later be extended to a more general model with multiple BSs in the JT set.

In Fig. 1, the baseband channel matrix between the $b$ th BS and the UE is denoted as $\mathbf{H}_{b} \in \mathbb{C}^{N_{R} \times N_{T}}$. The UE reports the precoder recommendation information either to each individual BS in the JT set or to the serving BS, which in turn exchanges this information among helper BSs over backhaul links. Let $\mathbf{W}_{b} \in \mathbb{C}^{N_{T} \times L}$ be the local precoding matrix of the $b$ th BS, where $L$ is the number of independent data substreams for the
UE. In addition, $\mathbf{W}_{b}$ is subjected to a per-BS power constraint $\operatorname{tr}\left\{\mathbf{W}_{b}^{\mathrm{H}} \mathbf{W}_{b}\right\} \leq P$, where $P$ is the maximum transmission power at each BS. Then, the signal received at the UE can be described by

$$
\mathbf{y}=\left[\mathbf{H}_{1}, \mathbf{H}_{2}\right]\left[\begin{array}{l}
\mathbf{W}_{1} \\
\mathbf{W}_{2}
\end{array}\right] \mathbf{x}+\mathbf{n}
$$

where $\mathbf{x}=\left[x_{1}, x_{2}, \ldots, x_{L}\right]^{\mathrm{T}}$ is the transmission data vector with $\mathbb{E}\left\{\mathbf{x x}^{\mathrm{H}}\right\}=\mathbf{I}_{L}$, and $\mathbf{n}$ is the noise vector with $\mathbb{E}\left\{\mathbf{n n}^{\mathrm{H}}\right\}=$ $\mathbf{R}_{n}$. Note that the interference from BSs outside of the interested JT set is incorporated into $\mathbf{n}$. Assuming that the interference is white colored [27], then $\mathbf{R}_{n}$ can be simplified to $\mathbf{R}_{n}=N_{0} \mathbf{I}_{N_{R}}$.

Suppose that a linear receiver $\mathbf{F} \in \mathbb{C}^{L \times N_{R}}$ is employed at the $\mathrm{UE}$ to detect $\mathbf{x}$. Then, the MSE of the $i$ th $(i \in\{1,2, \ldots, L\})$ detected substream can be represented by

$$
M_{i}=\mathbb{E}\left\{\left|\mathbf{F}_{i,:} \mathbf{y}-x_{i}\right|^{2}\right\} .
$$

In general, the average BER of the system is dominated by the substream with the maximum MSE [23]. Therefore, we want to jointly design $\mathbf{W}_{1}, \mathbf{W}_{2}$, and $\mathbf{F}$ to minimize the maximum of the substream MSEs. This MIN-MAX-MSE problem is formulated as

$$
\begin{array}{cl}
\min _{\mathbf{F}, \mathbf{W}_{1} \mathbf{W}_{2}} & \max \left\{M_{i} \mid i \in\{1,2, \ldots, L\}\right\} \\
\text { s.t. } & \operatorname{tr}\left\{\mathbf{W}_{b}^{\mathrm{H}} \mathbf{W}_{b}\right\} \leq P \forall b=1,2 .
\end{array}
$$

\section{B. Modeling of Imperfect Backhaul}

For full JT operations, the transmission data vector $\mathrm{x}$ needs to be available at the actual transmission points before the transmission starts. We assume that UE's data will always arrive at the serving BS from higher-layer entities in a timely and error-free manner. Then, the serving BS shares the data with the helper BSs by means of imperfect backhaul communications.

In this paper, we are mainly concerned with the impact of BS backhaul latency on system performance. In practice, backhaul links can be generally classified into three categories according to the physical media, i.e., optical fiber, copper (ADSL, ATM, VDSL, etc.), and wireless interface. The typical latency of the optical fiber is below $1 \mathrm{~ms}$, which can be neglected since the usual delay of CSI exchange and scheduling in cooperative MIMO systems is approximately $10 \mathrm{~ms}$ [15]. However, the latency of copper and wireless interface backhaul links varies from several milliseconds to tens of milliseconds, depending on the technology/standard. In addition, when the backhaul communication is based on a generic IP network, the backhaul latency also depends on the number of routers between two cooperative BSs and the topology of the network, e.g., star, ring, tree, mesh, etc. Furthermore, congestion in the routers causes an extra delay typically of several milliseconds [15].

Fig. 2 illustrates an example of a practical BS backhaul network, which combines the ring and tree topologies. If $\mathrm{BS}_{1}$ wants to share data with $\mathrm{BS}_{2}$, it has to set up a backhaul link over several routers. It should be noted that limited capacity 


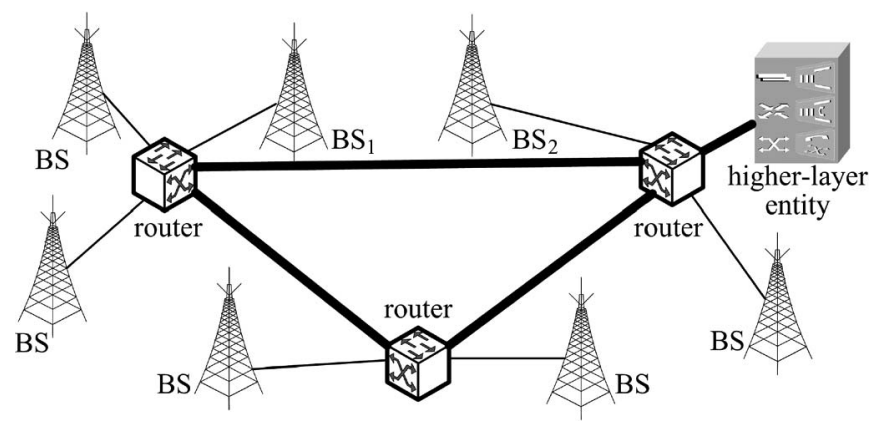

Fig. 2. Realistic BS backhaul network.

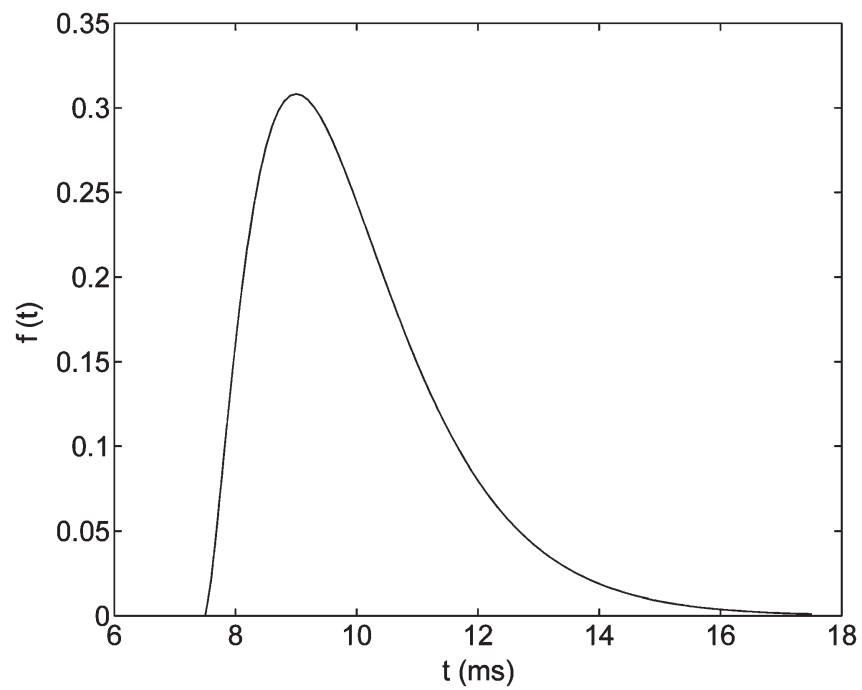

Fig. 3. Shifted gamma distribution of the backhaul delay.

is another important backhaul issue [16]. Most of the current cellular backhaul networks are designed for handover functions, which are not suited for data exchange in a large amount [3]. However, we assume capacity is adequate for backhaul communication throughout this paper.

According to [28], the maximum delay for normal backhaul links is around $20 \mathrm{~ms}$, and the typical average delay is expected to be within $10 \mathrm{~ms}$. A more detailed description of the backhaul latency model can be found in [22]. The backhaul delay conforms to a shifted gamma distribution [22], and its probability density function (pdf) can be represented by

$$
f(t)=\frac{\left(\frac{t-t_{0}}{\alpha}\right)^{\beta-1} \exp \left\{\frac{-\left(t-t_{0}\right)}{\alpha}\right\}}{\alpha \Gamma(\beta)}
$$

where $\alpha, \beta$, and $t_{0}$ are the scale, shape, and shift parameter, respectively, and $\Gamma(\cdot)$ denotes the gamma function. According to [22], the typical values are $\alpha=1, \beta=2.5$, and $t_{0}=7.5 \mathrm{~ms}$. The corresponding pdf curve is plotted in Fig. 3 for illustration purpose.

In every transmission slot, the $b$ th $(b \in\{2,3, \ldots, B\})$ helper BS joins JT with probability $p_{b}$, which is determined by the condition of the backhaul link between the serving BS and the $b$ th helper BS. In the following, $p_{b}$ will be referred to as participation probability, which can be computed from (4). Suppose that the JT operation is scheduled to be performed at a critical time $T$ after the serving cell pushes the UE's data into the backhaul network. Then, $p_{b}$ can be calculated as

$$
p_{b}=\int_{0}^{T} f(t) d t=\frac{\gamma\left(\beta, \frac{T-t_{0}}{\alpha}\right)}{\Gamma(\beta)}
$$

where $\gamma(\cdot, \cdot)$ is the lower incomplete gamma function [29]. In practice, $T$ will take a reasonably small value to avoid the performance degradation caused by outdated CSI. For instance, if $T=10 \mathrm{~ms}, p_{b} \approx 0.58$, and if $T=11 \mathrm{~ms}, p_{b} \approx 0.78$. Furthermore, if congestion occurs in the routers, $f(t)$ will suffer from an additional shift, i.e., $t_{0}$ will take large values. For instance, letting $t_{0}=8.5$, then if $T=10 \mathrm{~ms}, p_{b} \approx 0.3$, and if $T=11 \mathrm{~ms}$, $p_{b} \approx 0.58$.

\section{AdVAnCES AND DRAWbacks OF THE EXISTING SCHEMES}

In this section, we discuss the advances and drawbacks of the existing schemes, where different aspects such as system performance, required CSI feedback, and limited backhaul connectivity are carefully examined.

\section{A. GP Scheme}

The optimal precoding strategy for the full JT problem (3) is the GP, i.e., to view the distributed antenna ports from BSs in the JT set as a giant multiple-antenna system and generalize the well-studied point-to-point MIMO transmission strategies to JT across multiple BSs [1]. However, the difficulty lies in how to maintain the distributed per-BS power constraints while extending the point-to-point MIMO schemes to network MIMO ones. To our best knowledge, the MIN-MAX-MSE problem is still an open problem for the linear precoding design for JT network MIMO with distributed per-BS power constraints.

Hence, a sum power constraint is instead assumed for the GP to yield a lower bound for the MSE performance [1]. To formulate GP, rewrite (1) as

$$
\mathbf{y}=\mathbf{H W x}+\mathbf{n}
$$

where $\mathbf{H}$ and $\mathbf{W}$ denote the global channel matrix $\left[\mathbf{H}_{1}, \mathbf{H}_{2}\right]$ and global precoder $\left[\mathbf{W}_{1}^{\mathrm{T}}, \mathbf{W}_{2}^{\mathrm{T}}\right]^{\mathrm{T}}$, respectively. The MIN-MAX-MSE problem for GP can be reformulated from (3) as

$$
\begin{aligned}
\min _{\mathbf{F}, \mathbf{W}} & \max \left\{M_{i} \mid i \in\{1,2, \ldots, L\}\right\} \\
\text { s.t. } & \operatorname{tr}\left\{\mathbf{W}^{\mathrm{H}} \mathbf{W}\right\} \leq 2 P .
\end{aligned}
$$

Let $\mathbf{R}_{\mathbf{H}}=\mathbf{H}^{\mathrm{H}} \mathbf{R}_{n}^{-1} \mathbf{H}$, and its eigenvalue decomposition be

$$
\mathbf{R}_{\mathbf{H}}=\mathbf{V} \boldsymbol{\Lambda} \mathbf{V}^{\mathrm{H}}
$$

where $\mathbf{V} \in \mathbb{C}^{2 N_{T} \times 2 N_{T}}$ is a unitary matrix, and $\boldsymbol{\Lambda}=\operatorname{diag}\left\{\lambda_{i}\right\}$ is a semidefinite diagonal matrix, with diagonal entries $\lambda_{i} \mathrm{~s}$ being the eigenvalues of $\mathbf{R}_{\mathbf{H}}$. Then, the optimal solution for problem (7) is achieved by the joint linear transceiver design 
[30]. The optimal receiver should take the form of the Wiener filter [31] shown as

$$
\mathbf{F}^{\mathrm{opt}}=\mathbf{H}_{\mathrm{eq}}^{\mathrm{H}}\left(\mathbf{H}_{\mathrm{eq}} \mathbf{H}_{\mathrm{eq}}^{\mathrm{H}}+\mathbf{R}_{n}\right)^{-1}
$$

where $\mathbf{H}_{\mathrm{eq}}=\mathbf{H W}$ denotes the equivalent channel. Note that the Wiener filter has been proved to be the optimum linear receiver in the sense that it minimizes each of the substream MSEs [30]. Moreover, the optimal transmit precoding matrix $\mathbf{W}^{\text {opt }}$ should be

$$
\mathbf{W}^{\mathrm{opt}}=\tilde{\mathbf{W}} \mathbf{Q}^{\mathrm{H}}=\tilde{\mathbf{V}} \boldsymbol{\Sigma} \mathbf{Q}^{\mathrm{H}}
$$

where $\tilde{\mathbf{W}}=\tilde{\mathbf{V}} \boldsymbol{\Sigma}$, and the column of $\tilde{\mathbf{V}} \in \mathbb{C}^{2 N_{T} \times L}$ consists of the eigenvectors of $\mathbf{R}_{\mathbf{H}}$ corresponding to the $L$ largest eigenvalues in increasing order. The power loading matrix $\boldsymbol{\Sigma}=$ $\operatorname{diag}\left\{\sigma_{i}\right\}$. The $\sigma_{i}$ s are tuned so that the sum MSE with respect to $\mathbf{W}$ is minimized. In [30], it is proved that the optimal $\sigma_{i} \mathrm{~s}$ can be obtained by the famous waterfilling PA [31]

$$
\sigma_{i}=\sqrt{\left(\mu^{-1 / 2} \lambda_{i}^{-1 / 2}-\lambda_{i}^{-1}\right)^{+}}
$$

where $\mu^{-1 / 2}$ is the water level chosen to satisfy the power constraint with equality.

After minimizing the sum MSE by $\tilde{\mathbf{W}}$, a rotation operation is applied on it. In (10), $\mathbf{Q} \in \mathbb{C}^{L \times L}$ is a unitary rotation matrix such that all substream MSEs are equal. Thereby, the minimized sum MSE is equally divided for each substream, leading to a minimized MSE $\hat{M}$ for the maximum of the substream MSEs for problem (7), which can be concisely expressed as [30]

$$
\begin{aligned}
\hat{M} & =\max \left\{M_{i}\right\} \\
& =\frac{1}{L} \operatorname{tr}\left\{\left(\mathbf{I}_{L}+\left(\mathbf{W}^{\mathrm{opt}}\right)^{\mathrm{H}} \mathbf{R}_{\mathbf{H}} \mathbf{W}^{\mathrm{opt}}\right)^{-1}\right\} .
\end{aligned}
$$

Though the closed-form expression for $\mathbf{Q}$ does not exist, efficient algorithms to compute $\mathbf{Q}$ can be found in [32]. Note that (12) is a lower bound solution for the original problem (3) since the inter-BS PA implied by (10) is usually not the feasible solutions of (3). In addition, other practical issues such as limited backhaul and feedback overhead also compromise the performance of GP.

\section{B. AGP Scheme}

In practice, full JT, which is assumed by GP, is not always feasible due to backhaul limitations, such as overtime delay leading to incomplete or outdated data at the transmission points. Moreover, it is preferable for practical systems to have distributed schedulers due to considerations of low complexity and low cost, thereby some local scheduling constraints in helper BSs may also force them to temporarily leave the JT set and thus break the full JT operation. Hence, it is desirable to design a flexible JT scheme, in which the serving BS makes the JT scheduling decision and informs the helper BSs, and then the helper BSs can adaptively join or quit the upcoming JT operation according to their instantaneous states. If all the helper BSs are temporarily unavailable for JT, the system should be able to fall back to single-BS transmission (ST) smoothly. However, this gives rise to a feedback problem. The transmission assumption, based on which the recommended precoder is computed and fed back by the UE, may be inconsistent with the one when transmission eventually takes place. Consequently, the previously fed back precoder mismatches the transmission channel, causing performance degradation. This problem is not uncommon, particularly in 3GPP LTE-A systems [3]. A straightforward solution to the foregoing problem is to require the UE to feedback multiple precoder recommendations under different transmission assumptions. For example, in Fig. 1, the UE can feedback two precoders, one for JT and another for ST.

However, aside from the issue of necessary inter-BS signaling for switching between JT and ST precoders, additional feedback overhead incurred from multiple precoders will become very large, since the number of transmission assumptions can be as many as $2^{B-1}$. To avoid increasing the feedback overhead, another approach would be to instruct the UE to feedback the global precoder $\mathbf{W}^{\text {opt }}$ only. If any helper BSs are not ready for JT, they will mute themselves during the data transmission to keep the interference low, which is called dynamic point muting in the LTE-A system [33]. We assume that each helper BS is unaware of the states of the other helper BSs, and thus, they should stick to their respective subblock parts of $\mathbf{W}^{\mathrm{opt}}$. Such a scheme is hereafter referred to as AGP, and the corresponding precoder $\mathbf{W}_{b}^{\mathrm{opt}}$ for the $b$ th $\mathrm{BS}$ can be expressed as (13), shown at the bottom of the page. In (13), $\mathbf{W}_{i, \text { : }}$ denotes the $i$ th row of $\mathbf{W}$; thus, $\left[\mathbf{W}_{(b-1) N_{T}+1,:}^{\mathrm{opt} \mathrm{T}}, \ldots, \mathbf{W}_{b N_{T},:}^{\mathrm{opt} \mathrm{T}}\right]^{\mathrm{T}}$ represents the subblock part of $\mathbf{W}^{\text {opt }}$ spanning from the $\left((b-1) N_{T}+1\right)$ th row to the $\left(b N_{T}\right)$ th row of $\mathbf{W}^{\text {opt }}$. Since $\mathbf{W}^{\text {opt }}$ is optimized under the assumption of full JT, its subblock part shown in (13) may not match the individual $\mathbf{H}_{b}$ very well, which may result in large performance degradation when the system falls back to ST or partial JT. Therefore, in this paper, we propose a flexible and adaptive precoding scheme to alleviate the problem.

\section{Proposed Sequential and Incremental PRECODING SCHEME}

We propose a SIP scheme that is flexible and achieves satisfactory performance particularly for partial JT. In the proposed SIP scheme, the precoder optimization is performed for each helper BS according to the descending order of participation probabilities with the precoders of the previous BSs fixed. To facilitate the optimization, we require that the participation probabilities be determined by the serving BS based on (5),

$$
\mathbf{W}_{b}^{\text {opt }}= \begin{cases}\mathbf{0}, & \text { the } b \text { th BS is absent from JT } \\ {\left[\mathbf{W}_{(b-1) N_{T}+1,:}^{\text {opt T }}, \ldots, \mathbf{W}_{b N_{T},:}^{\text {opt T }}\right]^{\mathrm{T}},} & \text { otherwise }\end{cases}
$$


and the descending order of the participation probabilities be notified to the UE before it derives the precoders. We first investigate the precoder design for a two-BS JT network. The results are then extended to a multi-BS JT network.

\section{A. Precoder Design for the Two-BS JT Network}

We address the optimization problem (3) for the two-BS JT network introduced in Section II. We decouple problem (3) into two sequential steps.

In the first step, we optimize the precoder at the serving BS to ensure the service quality when the system falls back to ST. The problem can be formulated as

$$
\begin{aligned}
\min _{\mathbf{F}, \mathbf{W}_{1}} & \max \left\{M_{i} \mid i \in\{1,2, \ldots, L\}\right\} \\
\text { s.t. } & \operatorname{tr}\left\{\mathbf{W}_{1}^{\mathrm{H}} \mathbf{W}_{1}\right\} \leq P .
\end{aligned}
$$

Note that the foregoing problem is essentially the same as the problem (7) except for the substitution of $\mathbf{W}$ with $\mathbf{W}_{1}$ and the maximum power. Therefore, the optimal solution for (14) can be readily obtained as

$$
\mathbf{W}_{1}^{\mathrm{opt}}=\tilde{\mathbf{V}}_{1} \boldsymbol{\Sigma}_{1} \mathbf{Q}_{1}^{\mathrm{H}}
$$

where $\tilde{\mathbf{V}}_{1}, \boldsymbol{\Sigma}_{1}=\operatorname{diag}\left\{\sigma_{1, i}\right\}$, and $\mathbf{Q}_{1}$ are derived using the same method as their counterparts in (10) without subscripts.

In the second step, we proceed to optimize the performance of the two-BS JT with $\mathbf{W}_{1}$ fixed as $\mathbf{W}_{1}=\mathbf{W}_{1}^{\mathrm{opt}}$. This problem can be formulated as

$$
\begin{aligned}
\min _{\mathbf{F}, \mathbf{W}_{2}} & \max \left\{M_{i} \mid i \in\{1,2, \ldots, L\}\right\} \\
\text { s.t. } & \operatorname{tr}\left\{\mathbf{W}_{2}^{\mathrm{H}} \mathbf{W}_{2}\right\} \leq P .
\end{aligned}
$$

Problem (16) can be considered as a conditional optimization problem for $\mathbf{W}_{2}$ with the previously derived $\mathbf{W}_{1}^{\text {opt }}$ fixed. Direct optimization of problem (16) is not an easy task since the aforementioned approach of minimizing the sum MSE followed by unitary rotation for problems (7) and (14) cannot be applied here. Hence, we resort to an iterative method to minimize $\max \left\{M_{i}\right\}$.

Suppose that we have a precoding matrix $\mathbf{W}_{2}^{(n)}$ in the $n$th iteration. Then, the equivalent channel can be written as

$$
\mathbf{H}_{\mathrm{eq}}^{(n)}=\mathbf{H}_{1} \mathbf{W}_{1}^{\mathrm{opt}}+\mathbf{H}_{2} \mathbf{W}_{2}^{(n)} .
$$

Similar to (9), at the UE side, the Wiener filter is also employed to minimize the MSE, which is described as

$$
\mathbf{F}^{\mathrm{opt},(n)}=\mathbf{H}_{\mathrm{eq}}^{(n) \mathrm{H}}\left(\mathbf{H}_{\mathrm{eq}}^{(n)} \mathbf{H}_{\mathrm{eq}}^{(n) \mathrm{H}}+\mathbf{R}_{n}\right)^{-1} .
$$

Then, the MSE of the $i$ th substream can be written as

$$
M_{i}^{(n)}=\mathbb{E}\left\{\left|\mathbf{F}_{i,:}^{\mathrm{opt},(n)}\left(\mathbf{H}_{\mathrm{eq}}^{(n)} \mathbf{x}+\mathbf{n}\right)-x_{i}\right|^{2}\right\} .
$$

Denote the substream index associated with the maximum substream MSE as

$$
j(n)=\underset{i}{\arg \max }\left\{M_{i}^{(n)}\right\} .
$$

Next, we update $\left(\mathbf{W}_{2}^{(n+1)}\right)_{:, j(n)}$ subject to a substream power constraint $P_{2, j(n)}^{(n+1)}$ with fixed $\mathbf{F}^{\mathrm{opt},(n)}$ such that $M_{j(n)}^{(n+1)}$ is minimized. Denote $\mathbf{g}=\left(\mathbf{W}_{2}^{(n+1)}\right)_{:, j(n)}$ for convenience. Then, problem (16) can be formulated as

$$
\begin{array}{ll}
\min _{\mathbf{g}} & M_{j(n)}^{(n+1)}, \\
\text { s.t. } & \operatorname{tr}\left\{\mathbf{g}^{\mathrm{H}} \mathbf{g}\right\} \leq P_{2, j(n)}^{(n+1)}
\end{array}
$$

where $M_{j(n)}^{(n+1)}$ can be represented in detail as

$$
\begin{aligned}
M_{j(n)}^{(n+1)} & =\mathbb{E}\left\{\left|\mathbf{F}_{j(n),:}^{\mathrm{opt},(n)}\left(\left(\mathbf{H}_{1} \mathbf{W}_{1}^{\mathrm{opt}}+\mathbf{H}_{2} \mathbf{W}_{2}^{(n+1)}\right) \mathbf{x}+\mathbf{n}\right)-x_{j(n)}\right|^{2}\right\} \\
= & \sum_{i \neq j(n)}\left|\mathbf{F}_{j(n),:}^{\mathrm{opt},(n)}\left(\mathbf{H}_{1}\left(\mathbf{W}_{1}^{\mathrm{opt}}\right)_{:, i}+\mathbf{H}_{2}\left(\mathbf{W}_{2}^{(n+1)}\right)_{:, i}\right)\right|^{2} \\
& +\left|\mathbf{F}_{j(n),:}^{\mathrm{opt},(n)}\left(\mathbf{H}_{1}\left(\mathbf{W}_{1}^{\mathrm{opt}}\right)_{:, j(n)}+\mathbf{H}_{2} \mathbf{g}\right)\right|^{2} \\
& -2 \operatorname{Re}\left\{\mathbf{F}_{j(n),:}^{\mathrm{opt},(n)}\left(\mathbf{H}_{1}\left(\mathbf{W}_{1}^{\mathrm{opt}}\right)_{:, j(n)}+\mathbf{H}_{2} \mathbf{g}\right)\right\} \\
& +\mathbf{F}_{j(n),:}^{\mathrm{opt},(n)} \mathbf{R}_{\mathrm{n}}\left(\mathbf{F}_{j(n),:}^{\mathrm{opt},(n)}\right)^{\mathrm{H}}+N_{0} .
\end{aligned}
$$

We further fix $\left(\mathbf{W}_{2}^{(n+1)}\right)_{:, i}$ for $i \neq j(n)$. Omitting the irrelevant terms in (22) for simplicity, we get

$$
\begin{aligned}
M_{j(n)}^{(n+1)} & =\left|\mathbf{F}_{j(n),:}^{\mathrm{opt},(n)}\left(\mathbf{H}_{1}\left(\mathbf{W}_{1}^{\mathrm{opt}}\right)_{:, j(n)}+\mathbf{H}_{2} g\right)\right|^{2} \\
& -2 \operatorname{Re}\left\{\mathbf{F}_{j(n),:}^{\mathrm{opt},(n)}\left(\mathbf{H}_{1}\left(\mathbf{W}_{1}^{\mathrm{opt}}\right)_{:, j(n)}+\mathbf{H}_{2} \mathbf{g}\right)\right\} .
\end{aligned}
$$

Hence, problem (21) is equivalent to the following problem:

$$
\begin{array}{ll}
\min _{\mathbf{g}} & \tilde{M}_{j(n)}^{(n+1)} \\
\text { s.t. } & \operatorname{tr}\left\{\mathbf{g}^{\mathrm{H}} \mathbf{g}\right\} \leq P_{2, j(n)}^{(n+1)} .
\end{array}
$$

It is easy to verify that problem (24) is convex. Thus, we can obtain the optimal $\mathrm{g}$ from the Karush-Kuhn-Tucker (KKT) conditions [34]. The Lagrangian function of (24) is given by

$$
\mathcal{L}(\mathbf{g}, \eta)=\tilde{M}_{j(n)}^{(n+1)}+\eta\left(\operatorname{tr}\left\{\mathbf{g}^{\mathrm{H}} \mathbf{g}\right\}-P_{2, j(n)}^{(n+1)}\right)
$$

where $\eta \geq 0$ is the Lagrangian multiplier. Taking its derivative with respect to $\mathrm{g}^{*}$, we have

$$
\begin{array}{r}
\frac{\partial \mathcal{L}}{\partial \mathbf{g}^{*}}=\mathbf{H}_{2}^{\mathrm{H}}\left(\mathbf{F}_{j(n),:}^{\mathrm{opt},(n)}\right)^{\mathrm{H}} \mathbf{F}_{j(n),:}^{\mathrm{opt},(n)}\left(\mathbf{H}_{1}\left(\mathbf{W}_{1}^{\mathrm{opt}}\right)_{:, j(n)}+\mathbf{H}_{2} \mathbf{g}\right) \\
-\mathbf{H}_{2}^{\mathrm{H}}\left(\mathbf{F}_{j(n),:}^{\mathrm{opt},(n)}\right)^{\mathrm{H}}+\eta \mathbf{g .} \quad
\end{array}
$$

The KKT conditions are as follows:

$$
\left\{\begin{array}{l}
\frac{\partial \mathcal{L}}{\partial \mathbf{g}^{*}}=0, \\
\eta\left(\operatorname{tr}\left\{\mathbf{g}^{\mathrm{H}} \mathbf{g}\right\}-P_{2, j(n)}^{(n+1)}\right)=0, \\
\operatorname{tr}\left\{\mathbf{g}^{\mathrm{H}} \mathbf{g}\right\} \leq P_{2, j(n)}^{(n+1)}
\end{array}\right.
$$


From (27), the closed-form expression for $\mathbf{g}$ can be derived as

$$
\begin{aligned}
& \mathbf{g}=\left(\mathbf{H}_{2}^{\mathrm{H}}\left(\mathbf{F}_{j(n),:}^{\mathrm{opt},(n)}\right)^{\mathrm{H}} \mathbf{F}_{j(n),:}^{\mathrm{opt},(n)} \mathbf{H}_{2}+\eta \mathbf{I}\right)^{-1} \\
& \times \mathbf{H}_{2}^{\mathrm{H}}\left(\left(\mathbf{F}_{j(n),:}^{\mathrm{opt},(n)}\right)^{\mathrm{H}}-\left(\mathbf{F}_{j(n),:}^{\mathrm{opt},(n)}\right)^{\mathrm{H}} \mathbf{F}_{j(n),:}^{\mathrm{opt},(n)} \mathbf{H}_{1}\left(\mathbf{W}_{1}^{\mathrm{opt}}\right)_{:, j(n)}\right)
\end{aligned}
$$

where $\eta$ should be chosen such that (27b) and (27c) are satisfied.

The remaining problem is the PA strategy for $P_{2, j(n)}^{(n+1)}$. As shown in [30], the optimal solution for the MIN-MAX-MSE problem (7) is achieved when the substream MSEs are equal. Motivated by this result, we propose to transfer a small amount of power from the substream with minimum MSE to that with the maximum MSE in each iteration. In such a way, the maximum MSE $M_{j(n)}^{(n)}$ in the $n$th iteration will decrease to $M_{j(n)}^{(n+1)}$ due to the optimized precoding vector $\mathbf{g}$ together with the additional power bonus received from the substream with minimum MSE.

Letting

$$
k(n)=\underset{i}{\arg \min }\left\{M_{i}^{(n)}\right\}
$$

we update the PA as follows:

$$
\left\{\begin{array}{l}
P_{2, j(n)}^{(n+1)}=P_{2, j(n)}^{(n)}+\delta P_{2, k(n)}^{(n)} \\
P_{2, k(n)}^{(n+1)}=P_{2, k(n)}^{(n)} \times(1-\delta) \\
P_{2, i}^{(n+1)}=P_{2, i}^{(n)}, \quad \text { for } i \neq j(n), k(n)
\end{array}\right.
$$

where

$$
P_{2, l}^{(n)}=\left|\left(\mathbf{W}_{2}^{(n)}\right)_{:, l}\right|^{2}, l \in\{1,2, \ldots, L\}
$$

and $\delta$ is the percentage of power transferred from the $k(n)$ th substream to the $j(n)$ th substream. Based on (28) and (30), $\mathbf{W}_{2}^{(n+1)}$ can be updated as

$$
\left\{\begin{array}{l}
\left(\mathbf{W}_{2}^{(n+1)}\right)_{:, j(n)}=\mathbf{g} \\
\left(\mathbf{W}_{2}^{(n+1)}\right)_{:, k(n)}=\sqrt{1-\delta}\left(\mathbf{W}_{2}^{(n)}\right)_{:, k(n)} \\
\left(\mathbf{W}_{2}^{(n+1)}\right)_{:, i}=\left(\mathbf{W}_{2}^{(n)}\right)_{:, i}, \quad \text { for } i \neq j(n), k(n) .
\end{array}\right.
$$

Note that the PA should be initialized such that $\sum_{l=1}^{L} P_{2, l}^{(0)}=P$ so that in the following iterations the power constraint of $\mathbf{W}_{2}^{(n)}$ can always be satisfied. When the iterative algorithm converges, all substream MSEs should be equal. Hence, the termination criterion can be established based on the difference between $M_{j(n)}^{(n)}$ and $M_{k(n)}^{(n)}$. The proposed precoding scheme will be referred to as the SIP scheme hereafter and is summarized in Algorithm 1.

Algorithm 1 The SIP Scheme for Two-BS JT

Step 1: Compute $\mathbf{W}_{1}^{\text {opt }}$ according to (15);

Step 2: Obtain $\mathbf{W}_{2}^{\text {opt }}$ using the following iterative algorithm, 1) Initialization: Set $\mathbf{W}_{2}^{(1)}=\sqrt{P / L}\left[\mathbf{I}_{L}, \mathbf{0}_{L \times\left(N_{T}-L\right)}\right]^{\mathrm{T}}$ and $n=1$.

2) Iteration:

(a) Compute $\mathbf{H}_{\mathrm{eq}}^{(n)}$ and $\mathbf{F}^{\mathrm{opt},(n)}$ using (17) and (18), respectively;

(b) Use (28) to compute $\mathbf{g}$ and (32) to get $\mathbf{W}_{2}^{(n+1)}$;

3) Termination:

The algorithm terminates either when $M_{j(n)}^{(n)}$ and $M_{k(n)}^{(n)}$ converges, i.e., $\left(\left|M_{j(n)}^{(n)}-M_{k(n)}^{(n)}\right| / M_{j(n)}^{(n)} \leq \xi_{\text {th }}\right.$ or when $n \geq N_{\max }$, where $\xi_{\text {th }}$ is a predefined threshold and $N_{\max }$ is the maximum iteration number;

Output $\mathbf{W}_{2}^{\text {opt }}=\mathbf{W}_{2}^{(n)}$.

Else, $n=n+1$, and go to substep 2).

\section{B. Extension of the SIP Scheme}

In this section, we generalize our proposed SIP scheme to multi-BS scenarios, where $B>2$. Without loss of generality, the $B-1$ helper BSs are sorted by their participation probabilities in decreasing order as $p_{2} \geq p_{3} \geq \cdots \geq p_{B}$. Then, the corresponding precoders are sequentially and incrementally optimized with $\mathbf{W}_{2}$ first and $\mathbf{W}_{B}$ last based on Step 2 of the proposed SIP scheme. To be more specific, $\mathbf{W}_{b}(b \in\{2,3, \ldots, B\})$ is optimized with fixed $\mathbf{W}_{i}^{\mathrm{opt}}(i \in\{1,2, \ldots, b-1\})$ previously obtained from the SIP scheme. In addition, the equivalent channel shown in (17) now should be computed as

$$
\mathbf{H}_{\mathrm{eq}}^{(n)}=\sum_{i=1}^{b-1} \mathbf{H}_{i} \mathbf{W}_{i}^{\mathrm{opt}}+\mathbf{H}_{b} \mathbf{W}_{b}^{(n)} .
$$

If helper BS $b$ joins JT, its precoder will be $\mathbf{W}_{b}^{\text {opt }}$. Otherwise, it mutes its transmission.

To sum up, the proposed SIP scheme can be extended to be employed in a multi-BS JT scenario and is summarized in Algorithm 2.

Algorithm 2 The SIP Scheme for Multi-BS JT

Step 1: Compute $\mathbf{W}_{1}^{\text {opt }}$ according to (15);

$$
\text { Set } b=2 \text {; }
$$

Step 2: Obtain $\mathbf{W}_{b}^{\text {opt }}$ using the following iterative algorithm,

1) Initialization: Set $\mathbf{W}_{b}^{(1)}=\sqrt{P / L}\left[\mathbf{I}_{L}, \mathbf{0}_{L \times\left(N_{T}-L\right)}\right]^{\mathrm{T}}$ and $n=1 ;$

2) Iteration: 


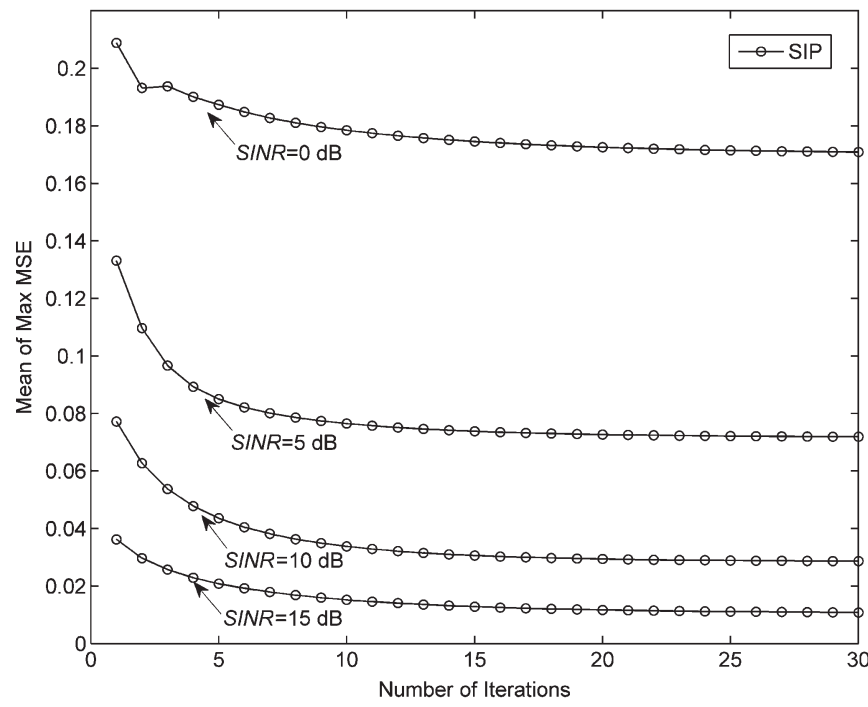

Fig. 4. Convergence of the SIP scheme for $B=2$ and $N_{R}=2$ (perfect feedback).

(a) Compute $\mathbf{H}_{\mathrm{eq}}^{(n)}$ and $\mathbf{F}^{\mathrm{opt},(n)}$ using (33) and (18), respectively;

(b) Use (28) to compute $\mathbf{g}$ and (32) to get $\mathbf{W}_{b}^{(n+1)}$;

3) Termination:

The algorithm terminates either when $M_{j(n)}^{(n)}$ and $M_{k(n)}^{(n)}$ converges, i.e., $\left(\left|M_{j(n)}^{(n)}-M_{k(n)}^{(n)}\right|\right) / M_{j(n)}^{(n)} \leq$ $\xi_{\text {th }}$ or when $n \geq N_{\max }$, where $\xi_{\text {th }}$ is a predefined threshold and $N_{\max }$ is the maximum iteration number; Output $\mathbf{W}_{b}^{\text {opt }}=\mathbf{W}_{b}^{(n)}$;

Else, $n=n+1$, then go to substep 2).

Step 3: If $b<B$, then $b=b+1$, and go to Step 2

Else, terminate the algorithm with $\mathbf{W}_{b}^{\text {opt }}(b \in$ $\{1,2, \ldots, B\})$ as the per-BS precoders.

\section{SIP Scheme With Codebook Based Feedback}

If a codebook, which is denoted as $\Omega$, is employed as the set of precoder candidates, then UE can exhaustively search $\Omega$, find the best precoder, and feedback its index to the BS using just a few bits. In practice, codebook-based feedback is commonly used in FDD systems such as the LTE-A system [3] due to low overhead costs. Here, we pursue the philosophy of SIP and propose the SIP scheme with codebook-based feedback.

For $\mathbf{W}_{1}$, the best precoder in the codebook $\Omega$ can be written as

$$
\mathbf{W}_{1}^{\text {opt,cb }}=\underset{\mathbf{W}_{1}^{\mathrm{cb}} \in \boldsymbol{\Omega}}{\arg \min } \max \left\{M_{i} \mid i \in\{1,2, \ldots, L\}\right\}
$$

where $M_{i}=\mathbb{E}\left\{\left|\mathbf{F}_{i,:}^{\text {opt }}\left(\mathbf{H}_{1} \mathbf{W}_{1}^{\mathrm{cb}} \mathbf{x}+\mathbf{n}\right)-x_{i}\right|^{2}\right\}$.

For $\mathbf{W}_{b}$, we fix the previously optimized precoders and incrementally find the best precoder for $\mathbf{W}_{b}$ from

$$
\mathbf{W}_{b}^{\mathrm{opt}, \mathrm{cb}}=\underset{\mathbf{W}_{b}^{\mathrm{cb}} \in \mathbf{\Omega}}{\arg \min \max }\left\{M_{i} \mid i \in\{1,2, \ldots, L\}\right\}
$$

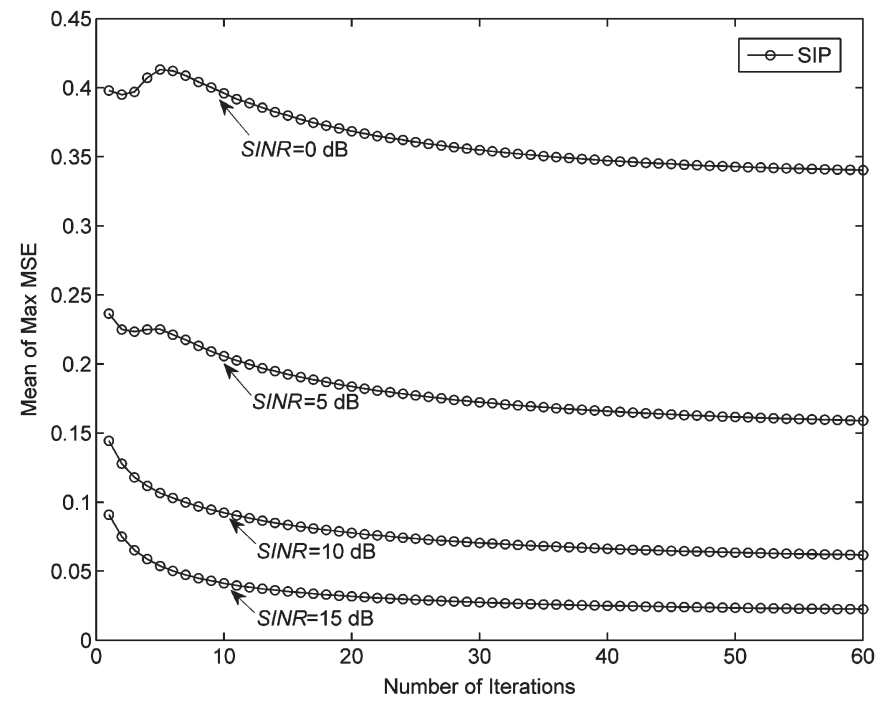

Fig. 5. Convergence of the SIP scheme for $B=2$ and $N_{R}=4$ (perfect feedback).

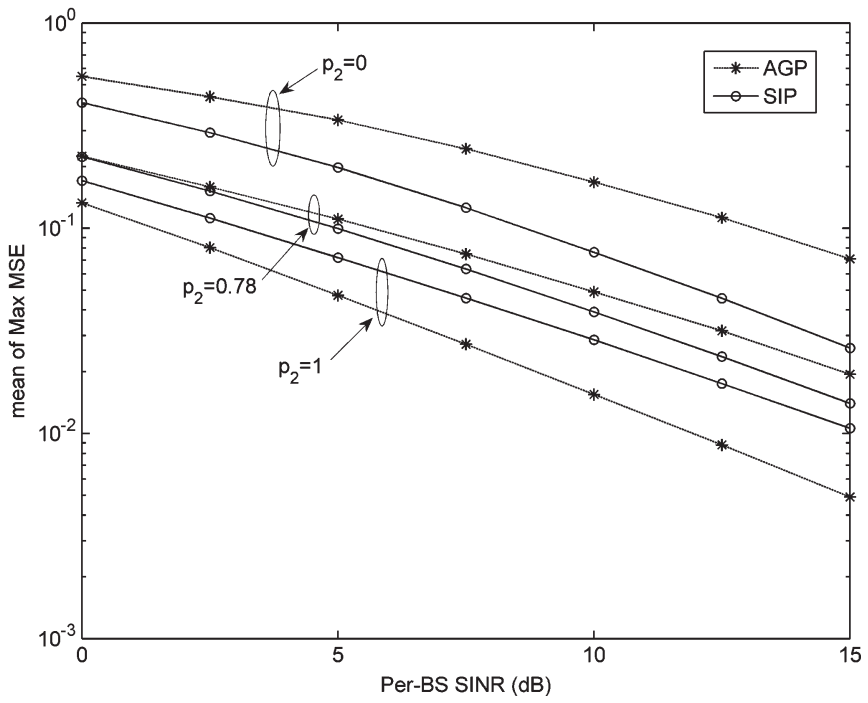

Fig. 6. Mean of the maximum of substream MSEs for $B=2$ and $N_{R}=2$ (perfect feedback).

where $\quad M_{i}=\mathbb{E}\left\{\left|\mathbf{F}_{i,:}^{\mathrm{opt}}\left(\mathbf{H}_{\mathrm{eq}} \mathbf{x}+\mathbf{n}\right)-x_{i}\right|^{2}\right\}, \quad$ and $\quad \mathbf{H}_{\mathrm{eq}}=$ $\sum_{i=1}^{b-1} \mathbf{H}_{i} \mathbf{W}_{i}^{\mathrm{opt}, \mathrm{cb}}+\mathbf{H}_{b} \mathbf{W}_{b}^{\mathrm{cb}}$.

In the AGP scheme, the best precoder from the global codebook $\Omega_{\mathrm{GP}}$ can be represented as

$$
\mathbf{W}^{\mathrm{opt}, \mathrm{cb}}=\underset{\mathbf{W}^{\mathrm{cb}} \in \boldsymbol{\Omega}_{\mathrm{GP}}}{\arg \min } \max \left\{M_{i} \mid i \in\{1,2, \ldots, L\}\right\}
$$

where $M_{i}=\mathbb{E}\left\{\left|\mathbf{F}_{i,:}^{\mathrm{opt}}\left(\mathbf{H} \mathbf{W}^{\mathrm{cb}} \mathbf{x}+\mathbf{n}\right)-x_{i}\right|^{2}\right\}$. Then, the $\mathbf{W}_{b}^{\mathrm{opt}, \mathrm{cb}} \mathrm{s}$ of the AGP scheme can readily be obtained from (13) with $\mathbf{W}^{\text {opt }}$ replaced by $\mathbf{W}^{\text {opt,cb }}$.

Suppose that the cardinality of $\Omega$ is $2^{d}$, then for each BS, $d$ bits are needed to feedback $\mathbf{W}_{b}^{\text {opt,cb }}$ from $2^{d}$ precoder candidates. To make a fair comparison between the SIP and AGP schemes, the cardinality of $\Omega_{\mathrm{GP}}$ should be $2^{B d}$, i.e., a total overhead of $B d$ bits are assumed for the feedback of precoders in both schemes. 


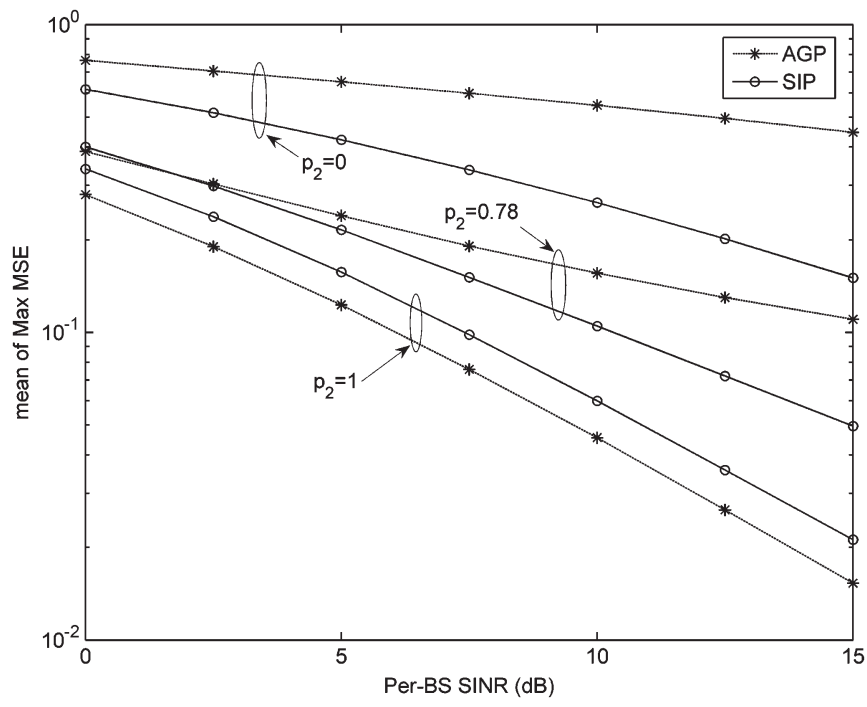

Fig. 7. Mean of the maximum of substream MSEs for $B=2$ and $N_{R}=4$ (perfect feedback).

\section{Simulation Results and Discussions}

In this section, we present simulation results to compare the maximum MSE and average BER performances of the proposed SIP scheme with those of the AGP scheme. We consider a practical setup where a single multiantenna UE with $N_{R}=2$ or 4 is served by a multi-BS JT set with $B=2$ or 3 and $N_{T}=4$. Suppose that the critical time $T=11 \mathrm{~ms}$ and $t_{0}$ for $\mathrm{BS}_{2}$ and $\mathrm{BS}_{3}$ are set to $t_{0,2}=7.5 \mathrm{~ms}$ and $t_{0,3}=8.5 \mathrm{~ms}$, respectively. As explained in Section II-B, the corresponding participation probabilities for $\mathrm{BS}_{2}$ and $\mathrm{BS}_{3}$ can be calculated using (5), and we get $p_{2} \approx 0.78$ and $p_{3} \approx 0.58$. Note that the water filling PA for the AGP and SIP schemes may lead to rank adaptation, i.e., dropping substreams with poor channel gains. For fairness, rank adaptation should be forbidden in our simulations. Therefore, when $N_{R}=4$, we fix $L=4$ to prevent rank adaptation and apply equal substream $\mathrm{PA}$ to $\mathbf{W}_{1}$, i.e., set $\sigma_{1, i}=P / L$ in (15), whereas the power transfer shown in (30) is applied for $\mathbf{W}_{b}$ s when $b \neq 1$. The water filling PA is only engaged for $\mathbf{W}_{1}$ in the case of $N_{R}=2$ when rank adaptation rarely happens. At the UE side, we assume that the Wiener filter is always employed.

We define per-BS signal to interference plus noise ratio (SINR) by SINR $=P / N_{0}$. All channels are assumed to experience uncorrelated Rayleigh fading, and the entries of $\mathbf{H}_{b}$ are independent identically distributed zero-mean circularly symmetric complex Gaussian random variables with unit variance. The results are averaged over 10000 independent channel realizations. As for the BER results, 1 million symbols obtained from the QPSK constellation are transmitted in each channel realization for each simulated SINR point. In addition, for the proposed SIP scheme, we set the convergence threshold $\xi_{\text {th }}=0.01$, power transfer percentage $\delta=1 \%$, and maximum iteration number $N_{\max }=100$.

\section{A. Convergence of SIP Scheme}

Before discussing the numerical results of the system performance, we first investigate the convergence behavior of the

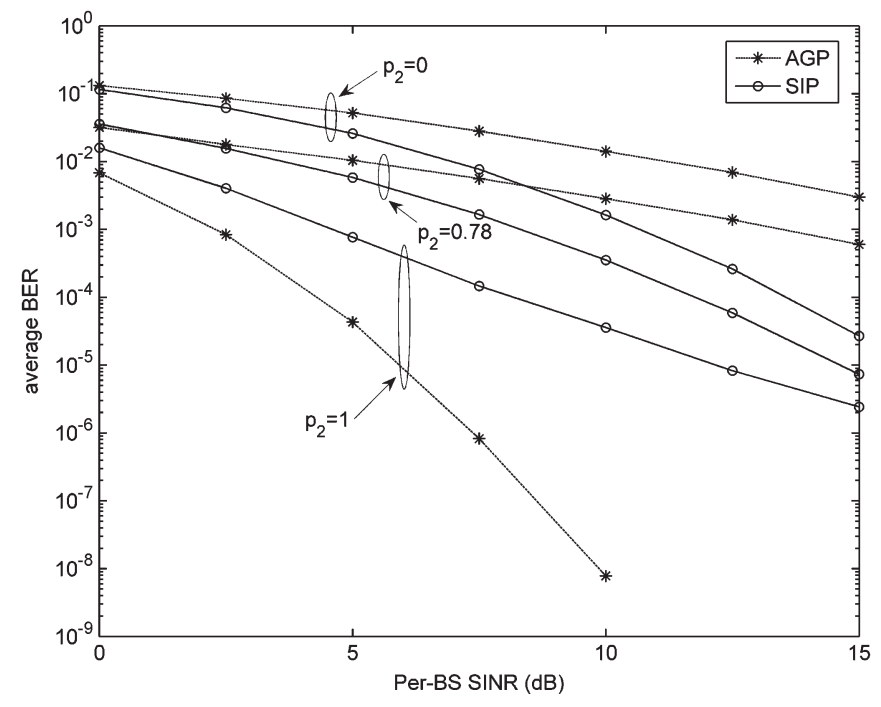

Fig. 8. Average BER for $B=2$ and $N_{R}=2$ (perfect feedback).

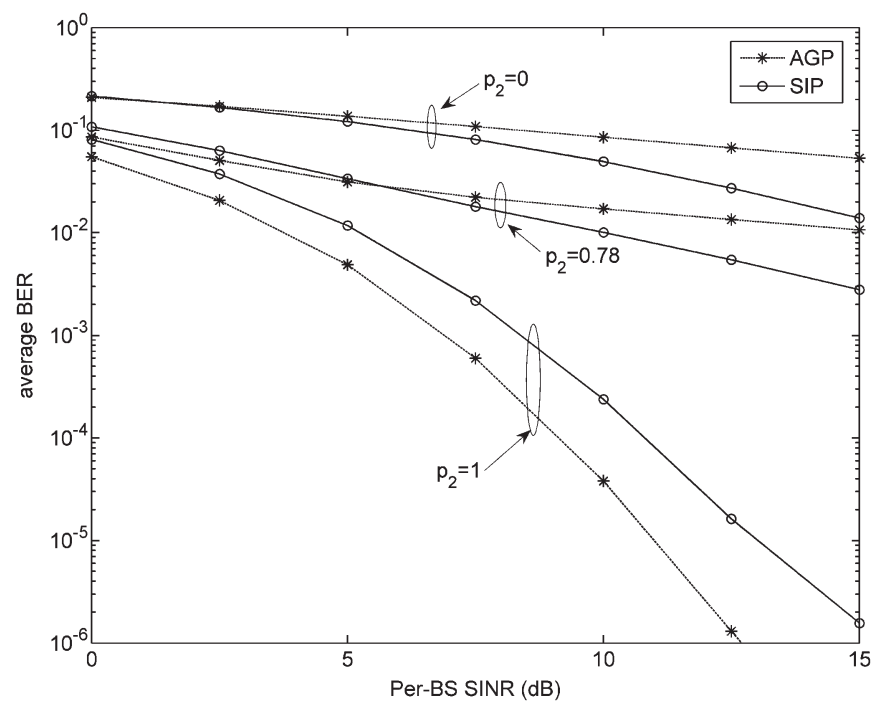

Fig. 9. Average BER for $B=2$ and $N_{R}=4$ (perfect feedback).

proposed SIP scheme summarized in Algorithm 1. Figs. 4 and 5 show the mean of the maximum of substream MSEs versus number of iterations for $B=2$ and $N_{R}=2$ or 4 with different SINRs. As seen from these two figures, the MSE always converges. When $N_{R}=2$, the MSE converges typically after 20 iterations, and more iterations are needed for the case of $N_{R}=4$. Moreover, the convergence of the SIP scheme for multi-BS JT is straightforward since the mathematical form of $\mathbf{H}_{\mathrm{eq}}^{(n)}$ in Algorithm 2 is essentially the same as that in Algorithm 1. Therefore, here we omit the illustration of algorithm convergence for the case of $B=3$.

\section{B. Performance of the Mean of the Maximum of Substream}

Figs. 6 and 7 show the average performance of the maximum of substream MSEs for $B=2$ and $N_{R}=2$ or 4 with different $p_{2}$. For the case of $p_{2}=0$, the system degenerates to ST due to broken backhaul, while the case of $p_{2}=1$ corresponds to full JT with perfect backhaul. As explained in Section III and 


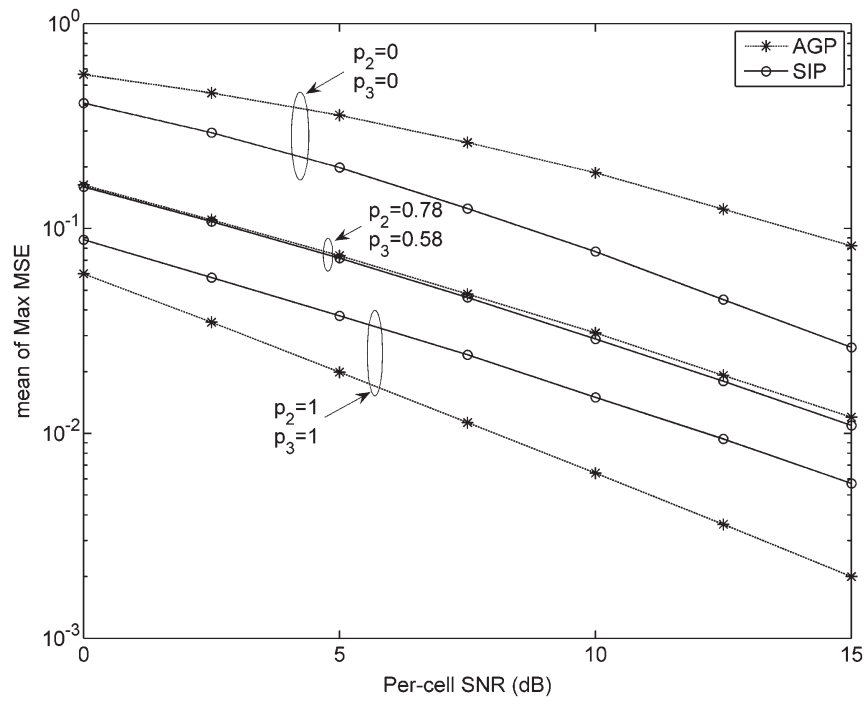

Fig. 10. Mean of the maximum of substream MSEs for $B=3$ and $N_{R}=2$ (perfect feedback).

observed in Figs. 6 and 7, the precoder for the AGP scheme is optimized under the assumption of full JT, which incurs large performance degradation when the system falls back to ST or partial JT. When the practical backhaul is considered, i.e., $p_{2}=0.78$, the proposed SIP scheme offers significant performance gain, and the gain is more pronounced in high SINR regimes because the sequentially and incrementally designed precoder matches the actual transmission channel better than the precoder in AGP that is optimized for full JT.

\section{Average BER Performance}

Figs. 8 and 9 show the average BER performance for $B=2$ and $N_{R}=2$ or 4 with different $p_{2}$. As seen from Figs. 8 and 9, our proposed SIP scheme also shows superior BER performance when $p_{2}=0.78$, particularly in high SINR regimes. When SINR $=15 \mathrm{~dB}$, compared with the AGP scheme, the proposed SIP scheme can reduce the average BER from $0.5 \times$ $10^{-3}$ to $10^{-5}$ and from $10^{-2}$ to $3 \times 10^{-3}$ for the case of $N_{R}=2$ and $N_{R}=4$, respectively.

\section{Performance of the Extended SIP Scheme}

For the extended case of $B=3$, we show the average performance of the maximum of substream MSEs in Figs. 10 and 11 and the corresponding BER results in Figs. 12 and 13 for $N_{R}=2$ or 4 with different $\left(p_{2}, p_{3}\right)$. Much like what we have observed previously, when the backhaul suffers from limited connectivity, e.g., $\left(p_{2}, p_{3}\right)=(0.78,0.58)$, the proposed SIP scheme significantly outperforms the AGP scheme in terms of average BER when the SINR is high. It is very interesting to note that the SIP and AGP schemes exhibit close averaged maximum MSE curves when $\left(p_{2}, p_{3}\right)=(0.78,0.58)$ in Fig. 10, but they have notable BER difference in favor of the SIP scheme in Fig. 12. One possible explanation might be that the overall MSE is also important to determine the BER performance, and the average MSE of the AGP scheme may not be well controlled as the maximum MSE. To investigate this issue, we conduct

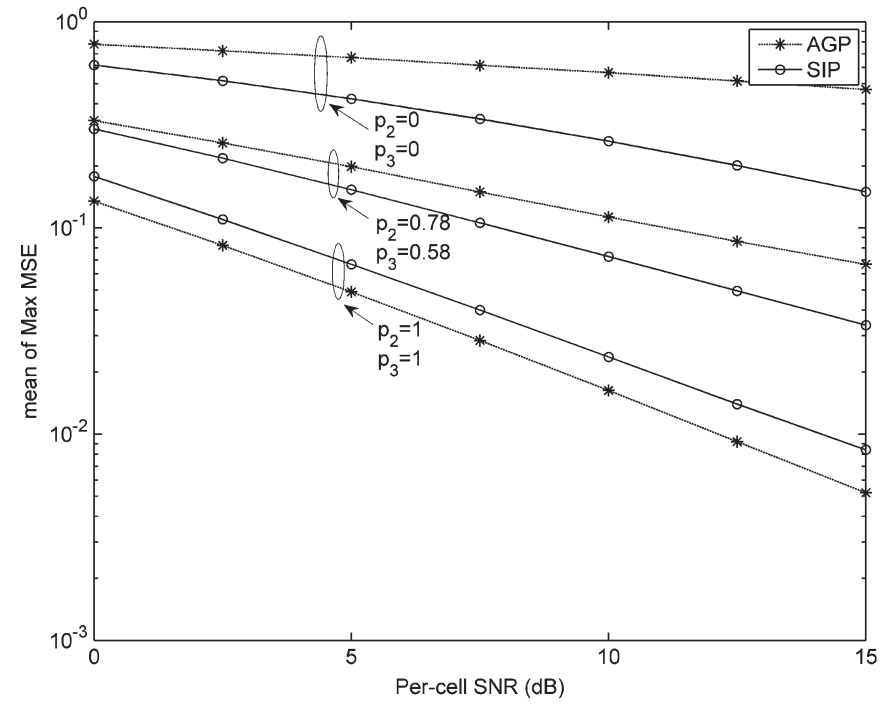

Fig. 11. Mean of the maximum of substream MSEs for $B=3$ and $N_{R}=4$ (perfect feedback).

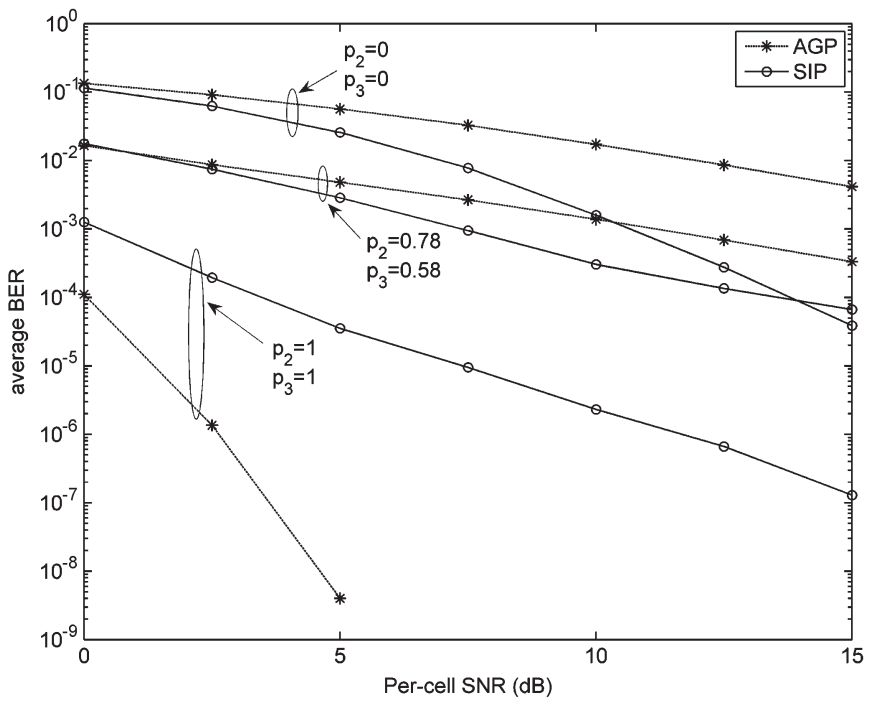

Fig. 12. Average BER for $B=3$ and $N_{R}=2$ (perfect feedback).

simulations to show the average MSE performance in Fig. 14 using parameters given in Fig. 10. From Fig. 14, we observe that the proposed SIP scheme does not outperform the AGP scheme in terms of average MSE, because the SIP scheme targets the optimization of the maximum substream MSE shown in problem (16), not the average MSE. Hence, the comparison of the average MSE performance does not highly relate to that of the BER performance. We suppose that the reason is that although the average maximum MSE performance is similar, the maximum substream MSE of the AGP scheme varies more widely than that of the SIP scheme, as can be observed from Fig. 10, where the variant range of the maximum substream MSE is roughly bounded by the "curves of mean of max MSE" for $\left(p_{2}, p_{3}\right)=(0,0)$ and $\left(p_{2}, p_{3}\right)=(1,1)$, and obviously, the variant range of SIP is narrower than that of AGP. The variant range of the maximum substream MSE indicates that the AGP scheme tends to generate a larger maximum substream MSE than the SIP scheme does in some poor cases, and the average 


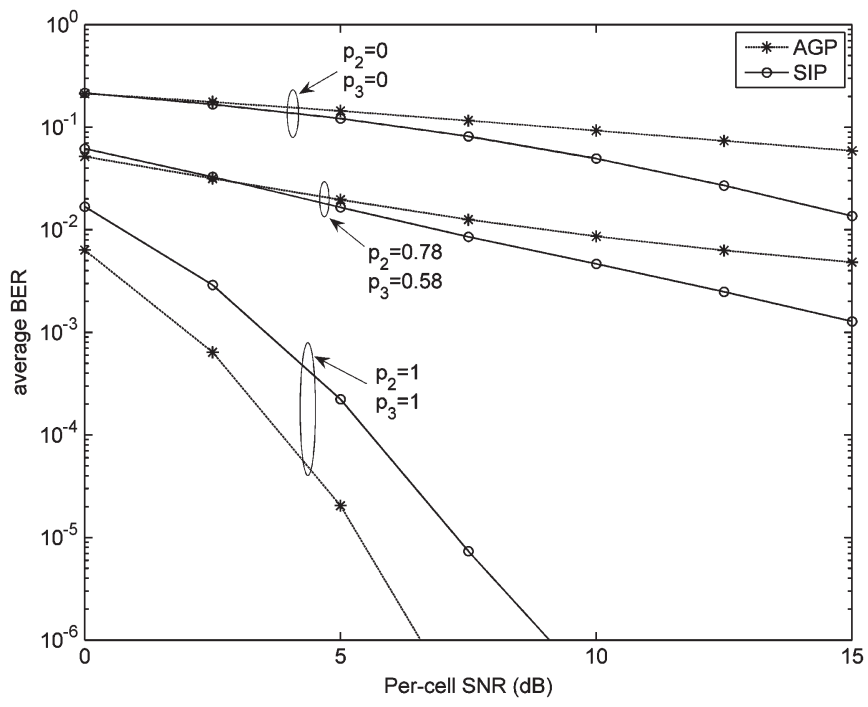

Fig. 13. Average BER for $B=3$ and $N_{R}=4$ (perfect feedback).

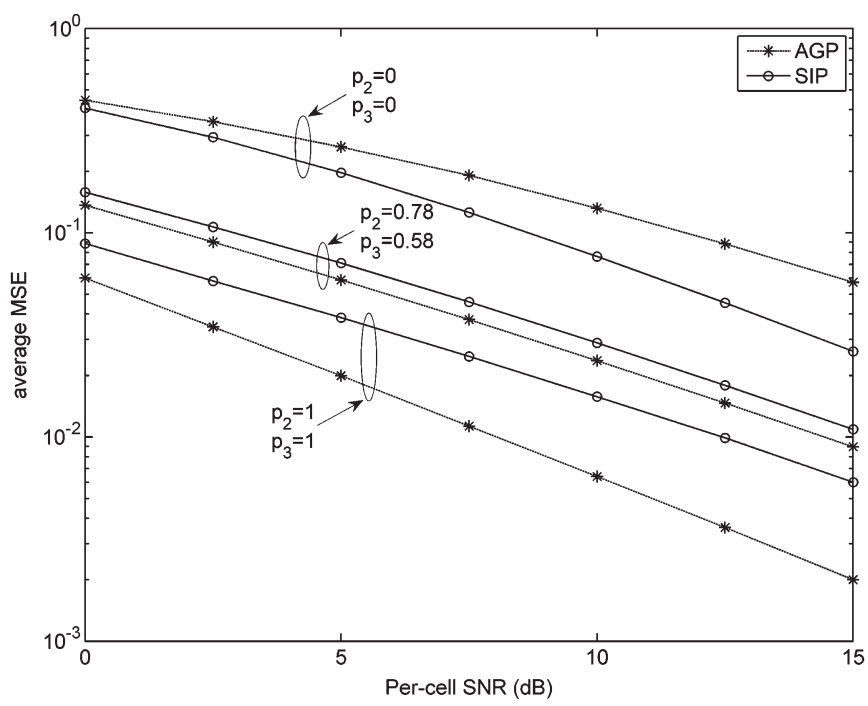

Fig. 14. Average MSE for $B=3$ and $N_{R}=2$ (perfect feedback).

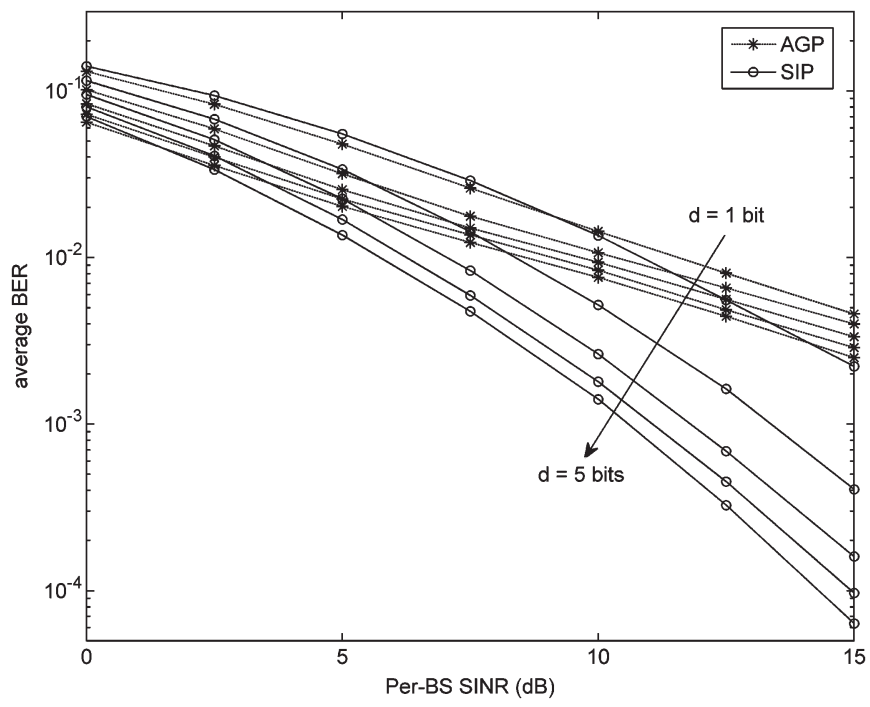

Fig. 15. Average BER for $B=2, N_{R}=2$, and $d=1 \sim 5$ (codebook-based feedback).

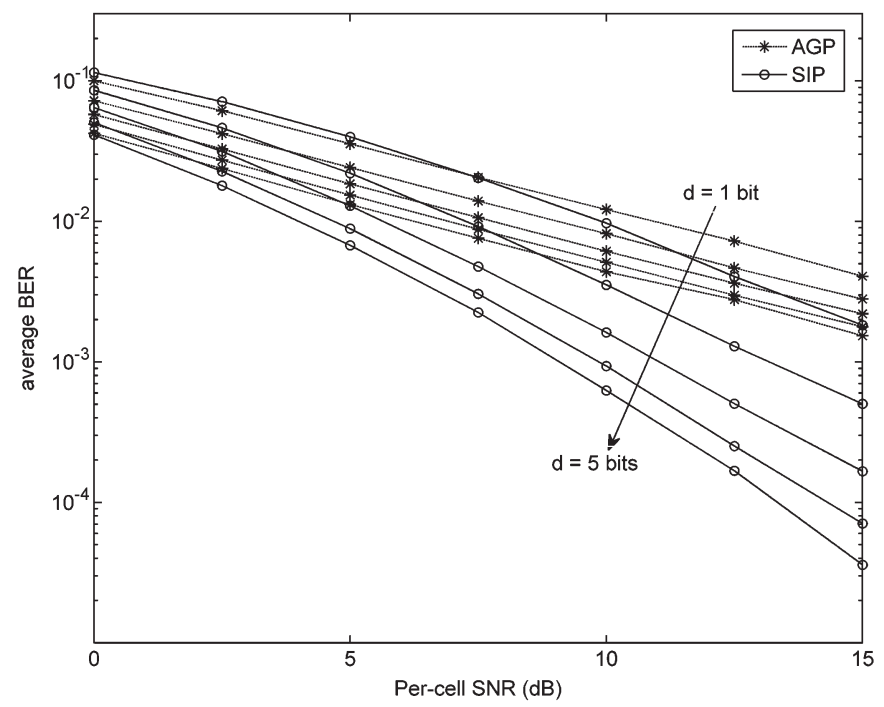

Fig. 16. Average BER for $B=3, N_{R}=2$, and $d=1 \sim 5$ (codebook-based feedback).

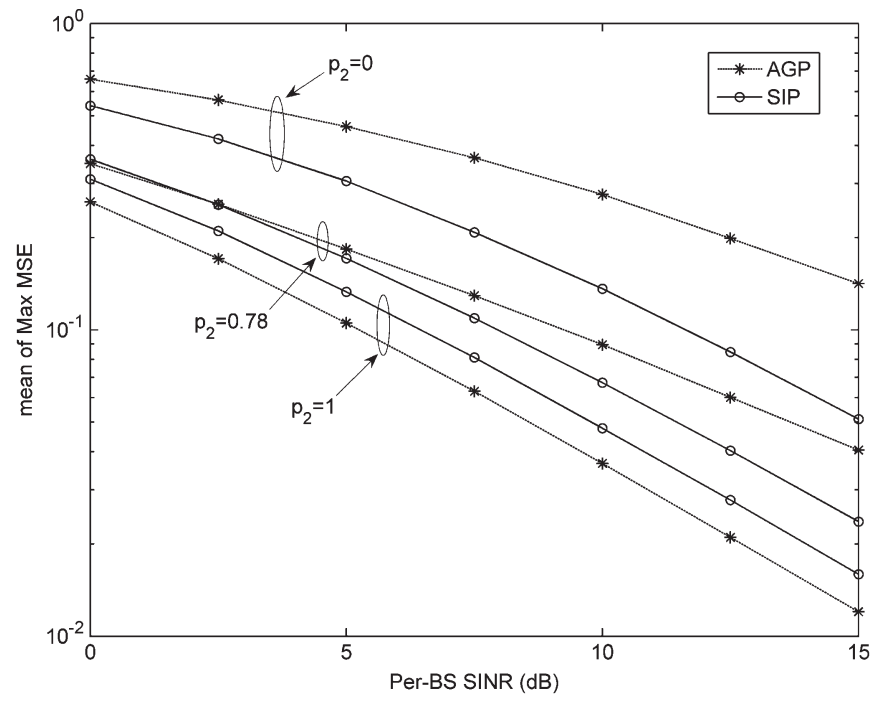

Fig. 17. Mean of the maximum of substream MSEs for $B=2, N_{R}=2$, and $d=4$ (codebook-based feedback)

BER performance is dominated by the large BERs resulted from poor-case maximum substream MSEs, which will lead to a higher BER performance for the AGP scheme.

\section{E. Performance of Finite Rate Feedback Systems}

Since the proposed SIP scheme is developed under the assumption of perfect feedback, it is highly motivated to investigate whether the SIP scheme still works in case of practical finite-rate feedback systems, i.e., the indices of the optimal codeword $\mathbf{W}_{b}^{\mathrm{opt}, \mathrm{cb}}$ s rather than $\mathbf{W}_{b}^{\mathrm{opt}}$ s themselves are fed back. First, we investigate the choice of $d$ in the codebook-based feedback. In Figs. 15 and 16, the average BER performances of the SIP and AGP schemes are shown for $B=2\left(p_{2}=0.78\right)$ or $B=3\left(\left(p_{2}, p_{3}\right)=(0.78,0.58)\right), N_{R}=2$, and $d=1 \sim 5$. We can observe in both figures that the performance gain suffers from a diminishing return as $d$ increases, and $d=4$ seems to be a good tradeoff between performance improvement and feedback overhead. Moreover, $d=4$ is a common assumption 


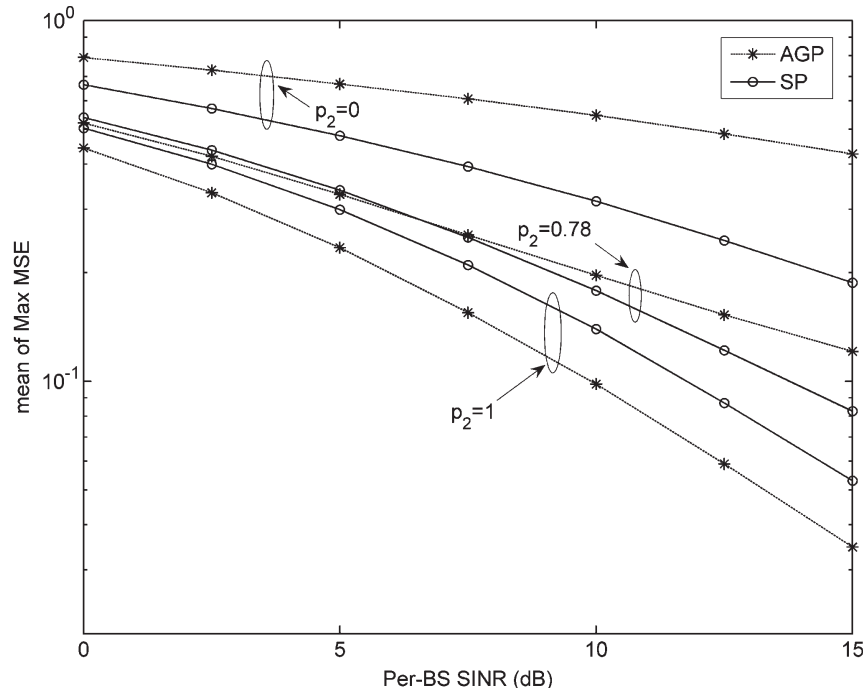

Fig. 18. Mean of the maximum of substream MSEs for $B=2, N_{R}=4$, and $d=4$ (codebook-based feedback).

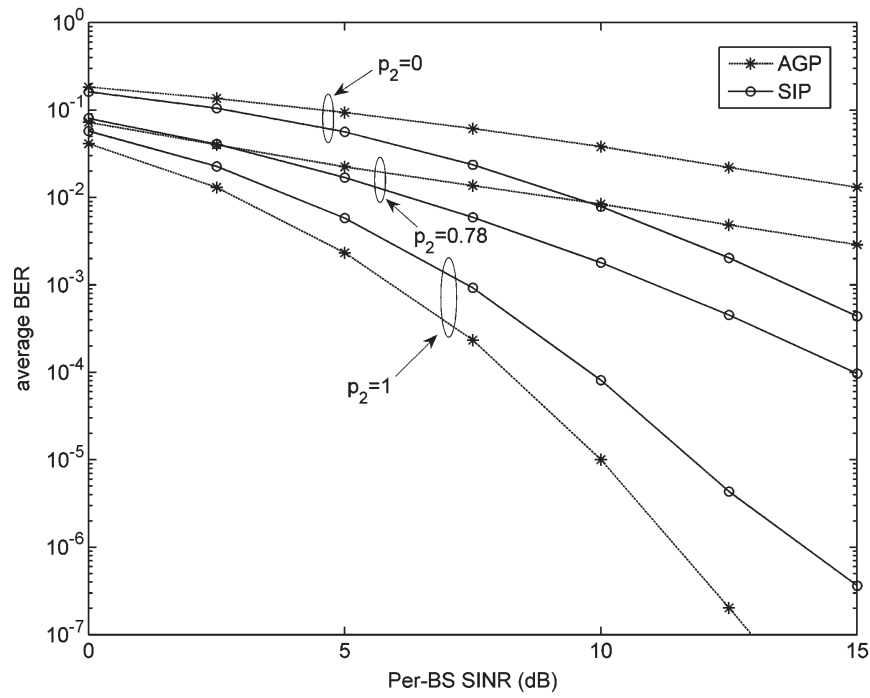

Fig. 19. Average BER for $B=2, N_{R}=2$, and $d=4$ (codebook-based feedback).

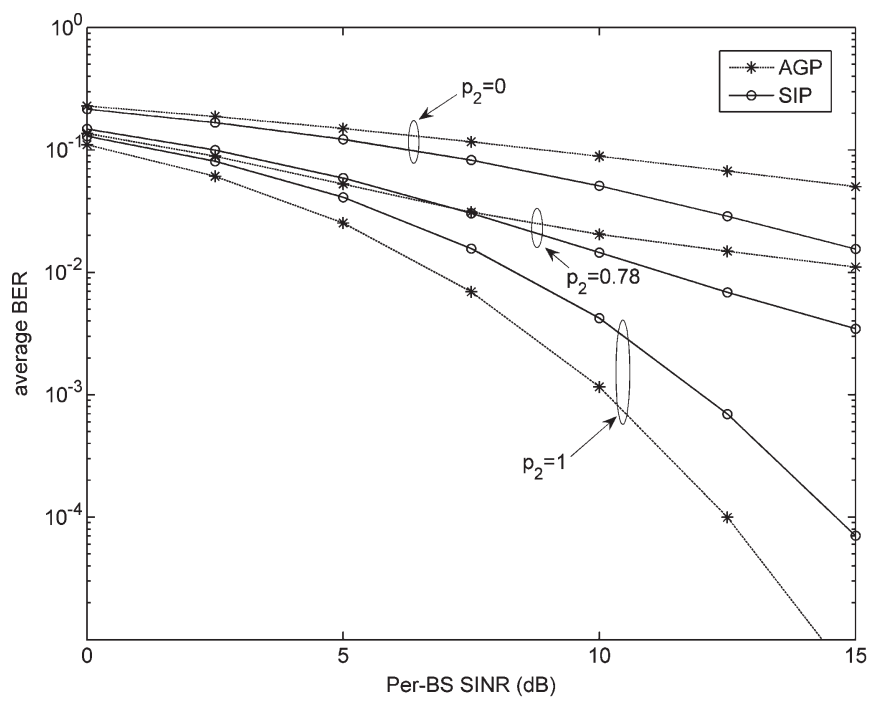

Fig. 20. Average BER for $B=2, N_{R}=4$, and $d=4$ (codebook-based feedback).

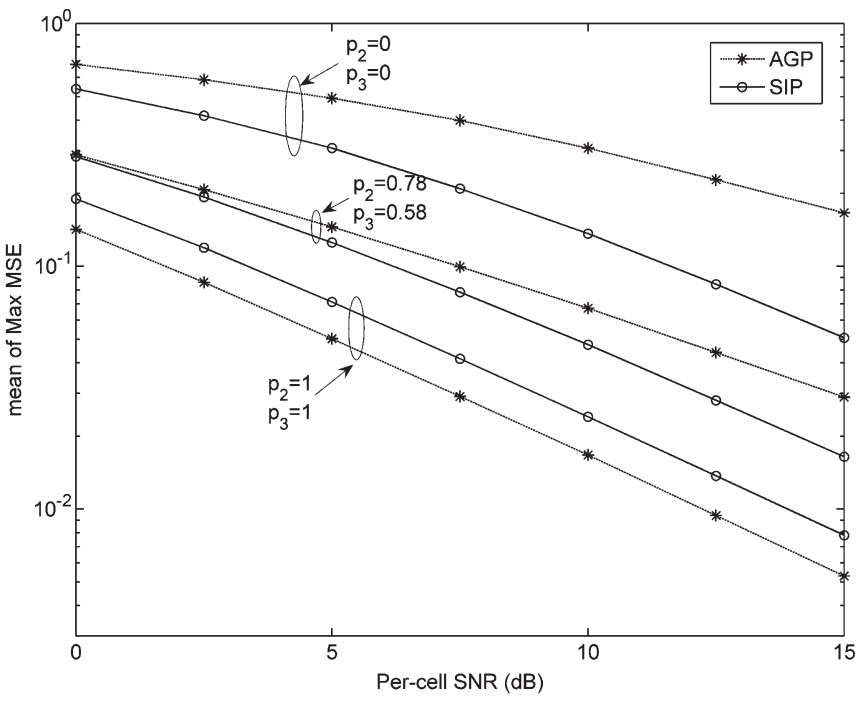

Fig. 21. Mean of the maximum of substream MSEs for $B=3, N_{R}=2$, and $d=4$ (codebook-based feedback).

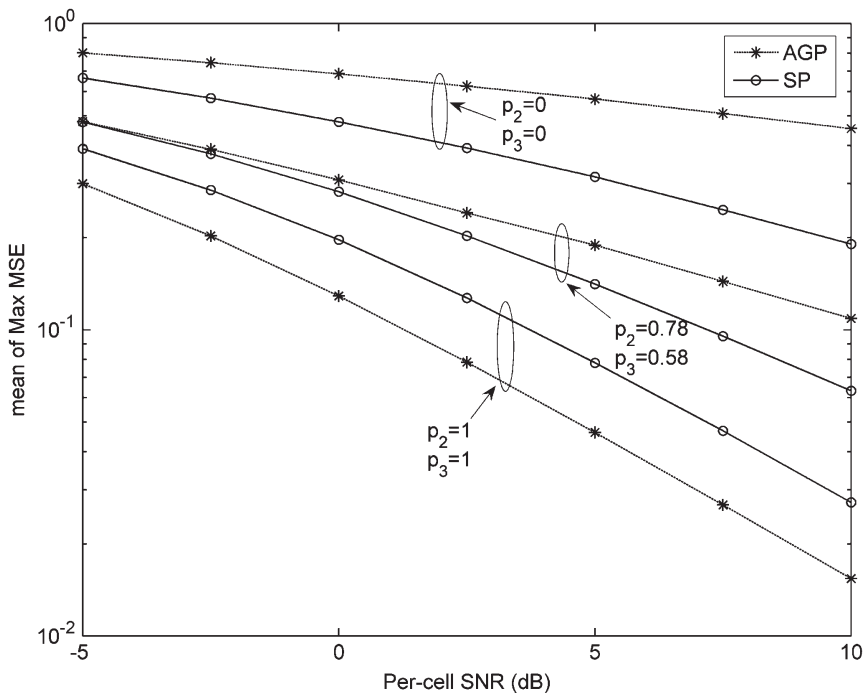

Fig. 22. Mean of the maximum of substream MSEs for $B=3, N_{R}=4$, and $d=4$ (codebook-based feedback).

for codebook designs for BSs with four transmit antennas in the LTE-A system [3]. Thus, in the following, we provide new simulation results plotted in Figs. 17-24 for $d=4$ to illustrate the performance degradation because of limited-bit feedback compared with Figs. 6-13. In our simulations, precoder candidates in the codebook are randomly generated as a matrix composed of orthogonal normalized vectors [35] for each channel realization. It can be observed from these figures that although the performance degradation is notable, the gains of the SIP scheme shown in Figs. 6-13 are safely preserved.

\section{F. Extension to the Multi-UE Scenario}

Finally, we briefly discuss the further extension of the proposed SIP scheme to the multi-UE scenarios and more sophisticated evaluations using system-level simulations. When multiple UEs are involved, the Wiener filter in (18) can be replaced by a block diagonal matrix to reflect that the individual UE's receive processing capability is captured by the 


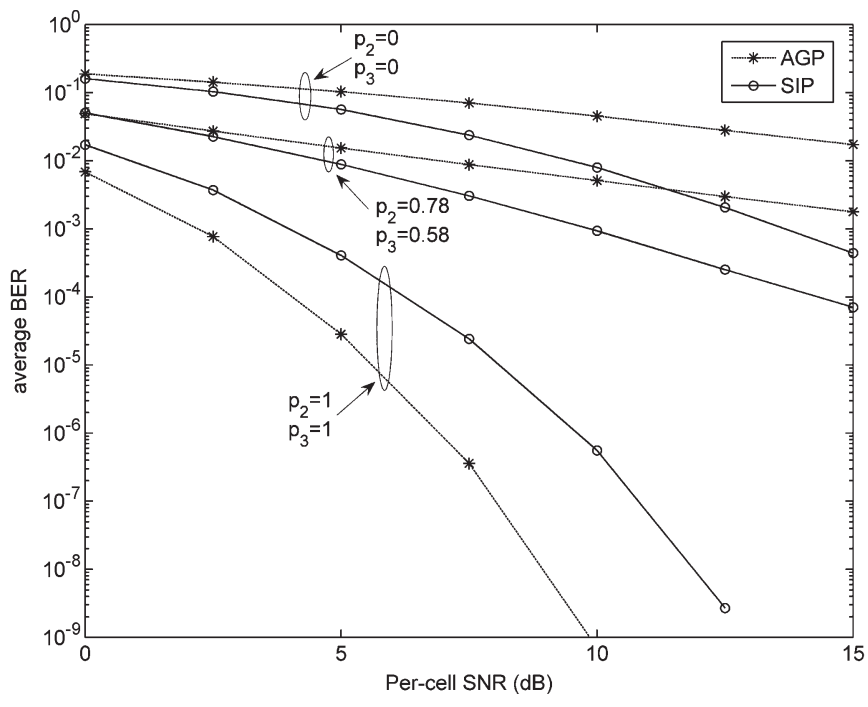

Fig. 23. Average BER for $B=3, N_{R}=2$, and $d=4$ (codebook-based feedback)

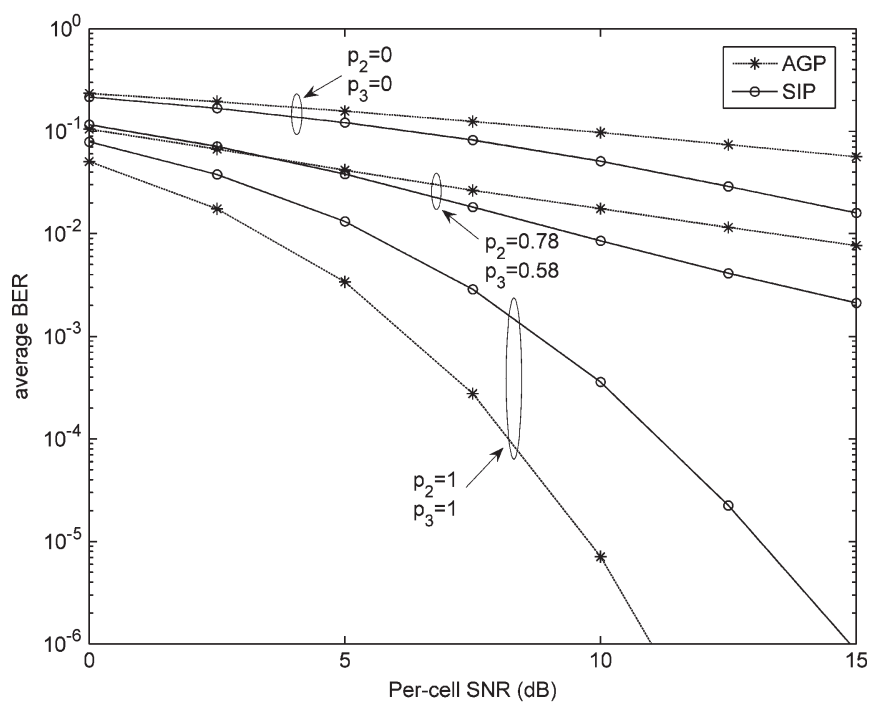

Fig. 24. Average BER for $B=3, N_{R}=4$, and $d=4$ (codebook-based feedback).

corresponding block matrix, and no receive cooperation is assumed among different UEs. Then, the precoders from multiple UEs can be derived using the proposed SIP scheme. However, the closed-form expression for $\mathbf{W}_{1}^{\text {opt }}$ shown by (15) no longer exists. The algorithms for optimizing $\mathbf{W}_{1}^{\mathrm{opt}}$ with various objective functions can be found in [23], which is beyond the scope of this paper and will be considered in the future works. In addition, the simulation results given in this paper are on a linklevel basis. More sophisticated evaluations using system-level simulations [33] considering cell-edge/cell-average spectral efficiency, UE distribution, multicell scheduler, retransmission in case of packet error, traffic modeling, propagation channel modeling, channel estimation errors, BS power settings, etc., are beneficial to the investigation of the performance gain offered by the proposed scheme in more practical scenarios. Thus, we will improve our simulation methods in the future works.

\section{CONCLUSION}

In this paper, transmission precoder design based on a sequential and incremental approach for JT network MIMO systems with imperfect backhaul has been studied. The conventional AGP scheme suffers from severe performance degradation in the event of partial JT and ST resulting from imperfect backhaul communications. An SIP scheme is proposed to overcome the drawbacks of the existing schemes. The key problem is first illustrated and solved with a two-BS JT system, and the results are then generalized to multi-BS JT network MIMO systems. Simulation results show that our scheme significantly outperforms the AGP scheme when a practical backhaul link is considered. Finally, future works extending the proposed SIP scheme to multi-UE scenarios and more sophisticated evaluations using system-level simulations are briefly discussed.

\section{REFERENCES}

[1] D. Gesbert, S. Hanly, H. Huang, S. Shamai, O. Simeone, and Y. Wei, "Multi-cell MIMO cooperative networks: A new look at interference," IEEE J. Sel. Areas Commun., vol. 28, no. 9, pp. 1380-1408, Dec. 2010.

[2] O. Somekh, O. Simeone, Y. Bar-Ness, A. M. Haimovich, and S. Shamai, "Cooperative multicell zeroforcing beamforming in cellular downlink channels," IEEE Trans. Inf. Theory, vol. 55, no. 7, pp. 3206-3219, Jul. 2009.

[3] "Further advancements for E-UTRA physical layer aspects," Tech. Rep. TR 36.814 V9.0.0, Mar. 2010.

[4] J. Zhang, R. Chen, J. G. Andrews, A. Ghosh, and R. W. Heath, "Networked MIMO with clustered linear precoding," IEEE Trans. Wireless Commun., vol. 8, no. 4, pp. 1910-1921, Apr. 2009.

[5] H. Dahrouj and W. Yu, "Coordinated beamforming for the multicell multiantenna wireless system," IEEE Trans. Wireless Commun., vol. 9, no. 5, pp. 1748-1759, May 2010.

[6] L. Venturino, N. Prasad, and X. Wang, "Coordinated linear beamforming in downlink multi-cell wireless networks," IEEE Trans. Wireless Commun., vol. 9, no. 4, pp. 1451-1461, Apr. 2010.

[7] C. B. Chae, S. Kim, and R. W. Heath, "Network coordinated beamforming for cell-boundary users: Linear and nonlinear approaches," IEEE J. Sel. Topics Signal Process., vol. 3, no. 6, pp. 1094-1105, Dec. 2009.

[8] S. Shamai and B. Zaidel, "Enhancing the cellular downlink capacity via co-processing at the transmitting end," in Proc. IEEE VTC, May 2001, vol. 3, pp. 1745-1749.

[9] N. Jindal, S. Vishwanath, and A. Goldsmith, "On the duality of Gaussian multiple-access and broadcast channels," IEEE Trans. Inf. Theory, vol. 50, no. 5, pp. 768-783, May 2004.

[10] W. Yu and T. Lan, "Transmitter optimization for the multi-antenna downlink with per-antenna power constraints," IEEE Trans. Signal Process., vol. 55, no. 6, pp. 2646-2660, Jun. 2007.

[11] M. H. M. Costa, "Writing on dirty paper," IEEE Trans. Inf. Theory, vol. IT-29, no. 3, pp. 439-441, May 1983.

[12] H. Huh, H. Papadopoulos, and G. Caire, "MIMO broadcast channel optimization under general linear constraints," in Proc. IEEE ISIT, Jun. 2009, pp. 2664-2668.

[13] D. P. Palomar and Y. Jiang, "MIMO transceiver design via majorization theory," Found. Trends Commun. Inf. Theory, vol. 3, no. 4, pp. 331-551, Nov. 2006.

[14] P. Marsch and G. Fettweis, "On downlink network MIMO under a constrained backhaul and imperfect channel knowledge," in Proc. IEEE Globecom, Nov. 2009, pp. 1-6.

[15] "Backhaul modelling for CoMP," presented at the 3GPP TSG-RAN1\#64 Meeting, Taipei, Taiwan, Feb. 2011, R1-111174.

[16] R. Zakhour and D. Gesbert, "Optimized data sharing in multicell MIMO with finite backhaul capacity," IEEE Trans. Signal Process., vol. 59, no. 12, pp. 6102-6111, Dec. 2011.

[17] "Final Report of 3GPP TSG RAN WG1 \#63bis v1.0.0," presented at the 3GPP TSG-RAN1\#64 Meeting, Taipei, Taiwan, Feb. 2011, R1-110611.

[18] "Final report of 3GPP TSG RAN WG1 \#64 v1.0.0," presented at the 3GPP TSG-RAN1\#65 meeting, Barcelona, Spain, May 2011, R1-111226.

[19] "Views on CoMP evaluation methodology," presented at the 3GPP TSGRAN1\#63bis Meeting, Dublin, Ireland, Jan. 2011, R1-110546. 
[20] “Revised SID proposal: Coordinated multi-point operation for LTE," presented at the 3GPP TSG RAN\#50 Meeting, Istanbul, Turkey, Dec. 2010, RP-101425.

[21] "Deployment and backhaul constraints for CoMP," in 3GPP TSGRAN1\#63bis Meeting, Dublin, Ireland, R1-110452, Jan. 2011.

[22] 802.20 Permanent Document 802.20 Evaluation Criteria (V1.0), Sep. 2005 802.20 Permanent Document.

[23] D. P. Palomar, M. A. Lagunas, and J. M. Cioffi, "On the optimal structure of transmit-receive linear processing for MIMO systems," in Proc. IEEE 40th Annu. Allerton Conf. Commun., Control, Comput., Oct. 2002, pp. 2-4.

[24] S. Zhou, J. Gong, Z. Niu, Y. Jia, and P. Yang, "A decentralized framework for dynamic downlink base station cooperation," in Proc. IEEE GLOBECOM, Dec. 2009, pp. 1-6.

[25] J. Moon and D. Cho, "Efficient cell-clustering algorithm for inter-cluster interference mitigation in network MIMO systems," IEEE Commun. Lett., vol. 15 , no. 3, pp. 326-328, Mar. 2011.

[26] "Discussion on the size of CoMP measurement set," presented at the 3GPP TSG RAN1\#67 Meeting, San Francisco, CA, Nov. 2011, R1114223.

[27] P. Skillermark, M. Almgren, D. Astely, M. Lundevall, and M. Olsson, "Simplified interference modeling in multi-cell multi-antenna radio network simulations," in Proc. IEEE VTC, May 2008, pp. 1886-1890.

[28] 3GPP TS 23.401 (V8.1.0), General Packet Radio Service (GPRS) enhancements for Evolved Universal Terrestrial Radio Access Network (E-UTRAN) access (Rel. 8), p. 129, Mar. 2008

[29] I. S. Gradshteyn and I. M. Ryzhik, Tables of Integrals, Series, and Products, 7th ed. New York: Academic, 2007.

[30] D. P. Palomar, J. M. Cioffi, and M. A. Lagunas, "Joint Tx-Rx beamforming design for multicarrier MIMO channels: A unified framework for convex optimization," IEEE Trans. Signal Process., vol. 51, no. 9, pp. 2381-2401, Sep. 2003.

[31] D. Tse and P. Viswanath, Fundamentals of Wireless Communication. Cambridge, U.K.: Cambridge Univ. Press, 2005.

[32] P. Viswanath and V. Anantharam, "Optimal sequences and sum capacity of synchronous CDMA systems," IEEE Trans. Inf. Theory, vol. 45, no. 6, pp. 1984-1991, Sep. 1999.

[33] 3GPP TR 36.819 (V11.0.0), Coordinated multi-point operation for LTE physical layer aspects (Rel. 11), Sep. 2011

[34] S. Boyd and L. Vandenberghe, Convex Optimization. Cambridge, U.K.: Cambridge Univ. Press, 2004

[35] W. Santipach and M. Honig, "Asymptotic capacity of beamforming with limited feedback," in Proc. IEEE ISIT, Jun. 2004, p. 289.

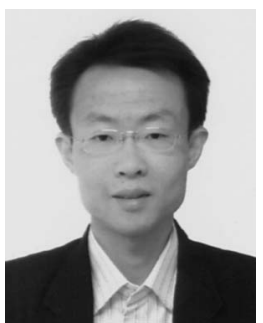

Ming Ding (M'11) received the B.S., M.S., and $\mathrm{Ph} . \mathrm{D}$. degrees in electronic engineering from Shanghai Jiaotong University, Shanghai, China, in 2004, 2007 , and 2012, respectively.

$\mathrm{He}$ is currently a Senior Researcher with Sharp Laboratories of China, Shanghai. He is also with the Department of Electronic Engineering, Shanghai Jiaotong University. His research interests include multiple-input-multiple-output-orthogonal frequency-division multiplexing (OFDM) technology, OFDM synchronization, relay systems, interference mitigation, cooperative communications, and wireless communication system modeling.

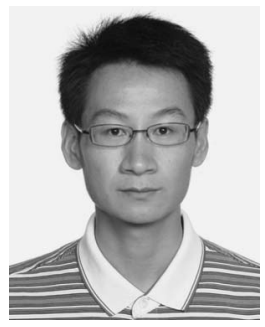

Jun Zou received the B.S. and M.S. degrees in electronic engineering from Shanghai Jiaotong University, Shanghai, China, in 2009 and 2012, respectively. $\mathrm{He}$ is currently working toward the Ph.D. degree in electrical and computer engineering with the Georgia Institute of Technology, Atlanta.

He is currently with the Department of Electronic Engineering, Shanghai Jiaotong University. His research interests include multiple-inputmultiple-output-orthogonal systems and relay communications.

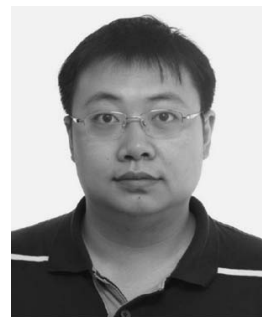

Zeng Yang received the B.S. degree from Nanjing University, Nanjing, China, in 2004 and the Ph.D. degree from the Chinese Academy of Sciences, Beijing, China, in 2009.

After graduation, he joined Sharp Labs of China, Shanghai, where he worked as a Senior Researcher His current research interests include radio resource allocation and optimization in the cellular networks, heterogeneous network architecture, and management.

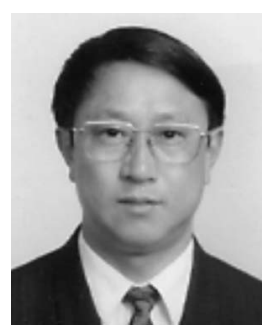

Hanwen Luo was born in 1950. He received the B.S. degree from Shanghai Jiaotong University, Shanghai, China, in 1977.

$\mathrm{He}$ is currently a Professor with Shanghai Jiaotong University, vice-director of the Shanghai Institute of Wireless Communications Technology, and vice-director of Institute of Wireless Communication Technology, Shanghai Jiaotong University. He was the leading specialist of the China 863 hightech program on Beyond Third-Generation wireless communication systems and the China 973 high-tech program on the research of military equipment. He has published more than 60 papers in IEEE journals and conferences. His research interests include mobile and personal communications.

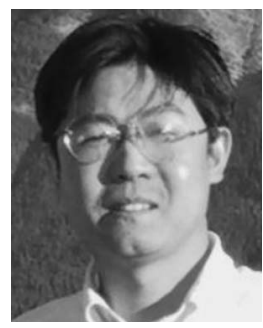

Wen Chen (M'03-SM'11) received the B.S. and M.S. degrees from Wuhan University, Wuhan, China, in 1990 and 1993, respectively and the Ph.D. degree from the University of Electro-Communications, Tokyo, Japan, in 1999.

He was a Researcher with the Japan Society for the Promotion of Sciences from 1999 to 2001. In 2001, he joined the University of Alberta, Edmonton, $\mathrm{AB}$, Canada, starting as a Postdoctoral Fellow with the Information Research Laboratory and continuing as a Research Associate with the Department of Electrical and Computer Engineering. Since 2006, he has been a Full Professor with the Department of Electronic Engineering, Shanghai Jiaotong University, Shanghai, China, where he is also the Director of the Institute for Signal Processing and Systems. He has published more than 100 papers in IEEE journals and conferences. His interests cover network coding, cooperative communications, cognitive radio, and multiple-input-multiple-output orthogonal frequency-division multiplexing systems.

Dr. Chen received the Ariyama Memorial Research Prize in 1997 and the PIMS Post-Doctoral Fellowship in 2001. He received the honors of "New Century Excellent Scholar in China" in 2006 and the "Pujiang Excellent Scholar in Shanghai" in 2007. He was elected to the Vice General Secretary of the Shanghai Institute of Electronics in 2008. He is on the editorial board of the International Journal of Wireless Communications and Networking and serves on the Journal of Communications, Journal of Computers, Journal of Networks, and the EURASIP Journal on Wireless Communications and Networking as (lead) Guest Editor. He was the Technical Program Committee Chair for the IEEE International Conference on Circuits and Systems for Communications in 2008, the General Conference Chair for the IEEE International Conference on Computer and Information Science in 2009, and the International Conference on Wireless Communications, Networking, and Information Security in 2010. 